\title{
Capacity-achieving CPM schemes
}

\author{
Alberto Perotti, Member, IEEE, Alberto Tarable, Member, IEEE, \\ Sergio Benedetto, Fellow, IEEE, and Guido Montorsi, Senior Member, IEEE, \\ Politecnico di Torino, Dipartimento di Elettronica \\ Corso Duca degli Abruzzi, 24 \\ I-10129 - Torino (Italy) \\ E-mail: \{alberto.perotti, alberto.tarable, \\ sergio.benedetto, guido.montorsi\}@polito.it
}

\begin{abstract}
The pragmatic approach to coded continuous-phase modulation (CPM) is proposed as a capacity-achieving lowcomplexity alternative to the serially-concatenated CPM (SCCPM) coding scheme. In this paper, we first perform a selection of the best spectrally-efficient CPM modulations to be embedded into SC-CPM schemes. Then, we consider the pragmatic capacity (a.k.a. BICM capacity) of CPM modulations and optimize it through a careful design of the mapping between input bits and CPM waveforms. The so obtained schemes are cascaded with an outer serially-concatenated convolutional code to form a pragmatic coded-modulation system. The resulting schemes exhibit performance very close to the CPM capacity without requiring iterations between the outer decoder and the CPM demodulator. As a result, the receiver exhibits reduced complexity and increased flexibility due to the separation of the demodulation and decoding functions.
\end{abstract}

\section{INTRODUCTION}

Continuous phase modulation (CPM) is a class of bandwidth efficient modulation schemes [1] whose characteristic of constant envelope makes them robust with respect to the nonlinearities introduced by the analog baseband and radiofrequency sections of low-cost transceivers usually found in consumer-type communications equipment. Good application examples are the second generation GSM cellular system, and satellite communication systems.

A CPM modulator is a finite-state machine delivering to the channel a continuous-phase, constant envelope waveform that depends on its input symbol and internal state. In [2] a CPM modulator has been shown to be decomposable into the cascade of a time-invariant convolutional encoder (continuousphase encoder, CPE) operating on a ring of integers, and of a time-invariant memoryless modulator (MM). Recently, this decomposition has been exploited by inserting an outer convolutional encoder whose coded bits enter an interleaver and then the CPE, thus forming what is known in the literature as a serially-concatenated convolutional encoder (SCCC) [3]. In the following, we will call this scheme SC-CPM.

Iterating between the outer encoder and the CPE through the interleaver yields rather good performance [4], [5], [6], which should be compared with the capacity of the CPM scheme. Recently, the authors of [7] proposed a simulation-based method for the computation of the capacity of channels with memory, which can be applied to the evaluation of the capacity of the CPM schemes. A similar method has been proposed in
[8], where it has been applied to a generalized form of CPM modulation achieving improved spectral efficiency.

In communication systems where the channel conditions can vary significantly with time, an efficient radio resource management requires the availability at the physical layer of adaptive coding-modulation, capable of varying its characteristics of bandwidth and energy efficiency following the channel rate of variation. This requirement has originated an active research on bit-interleaved (also known as pragmatic) coded modulation [9], [10], [11]. Pragmatic coded modulation consists in cascading a highly performing versatile binary encoder (typically, a punctured turbo or low-density paritycheck code) with several modulation schemes with increasingly large signal alphabets. The versatile encoder is capable of varying both its rate and codeword length in a wide range in order to achieve increasing spectral efficiencies. An example of the obtainable results has been published in [12], which demonstrates a scheme based on SCCC and linear twodimensional modulations yielding spectral efficiencies in a very wide range lying around $1 \mathrm{~dB}$ from the Shannon capacity limits. This paper deals with the extension of the pragmatic approach to CPM modulation (called P-CPM in the following). This approach does not require iterations between the outer encoder and the CPE, since the CPM is treated exactly as a linear modulation in a bit-interleaved turbo-trellis coded modulation. A nice consequence is that the overall CPE state complexity is not enhanced by the number of iterations, thus permitting to increase the bandwidth efficiency through the use of a larger number of CPE states.

In [13] a first attempt to design pragmatic schemes employing CPM modulation has been presented. The authors showed that the pragmatic capacity of CPM schemes heavily depends on the mapping between information bits and CPM signals, and presented a simulation-based mapping optimization algorithm with some examples. Further improvements have been presented in [14], where a procedure for optimizing such mapping has been proposed. The optimized CPM modulators have been embedded into a P-CPM scheme and its performance has been assessed through simulation. For the considered CPM schemes, performance improvements of more than $2 \mathrm{~dB}$ in pragmatic capacity have been observed.

Previuos literature on the subject includes [15], where a scheme consisting of a parallel concatenated turbo code and continuous-phase modulation has been investigated and 
a modified encoder has been proposed. In [16], CPM modulations have been studied in the context of multiple antenna systems with layered space-time coding. Reduced-complexity receivers have been proposed and differential encoding has been introduced in order to obtain an increased coding gain.

This paper presents a systematic and comprehensive approach to the problem of designing capacity approaching SCCPM and P-CPM, through the evaluation of the CPM and P-CPM capacities, the search for optimal (in the sense of offering the best trade-off between bandwidth efficiency, energy efficiency and complexity) CPM modulations, their embedding into SC-CPM and P-CPM schemes through the optimization of the mapping between coded bits and CPM waveforms, and a thorough comparison of their error probability performance with respect to capacities. Examples refer to rectangular and raised cosine frequency waveforms, and to three spectral efficiencies deemed important for the applications.

This paper is organized as follows: in Sec. II a general description of the considered coding and modulation systems is given. Sec. III presents the procedure used for the CPM capacity computation through simulation. In Sec. IV the optimization procedure used to select the best CPM parameters to be used in SC-CPM schemes is defined. Sec. V presents the procedure used for the optimization of P-CPM schemes: first, the optimal mapping is derived, the corresponding optimized CPE scheme is given and then the CPM schemes with best pragmatic capacity are selected. Sec. VI shows how the optimized CPM schemes have been embedded into SC-CPM and P-CPM coded systems. Finally, Sec. VII shows the results obtained using the selected CPM modulations in SC-CPM and P-CPM schemes.

\section{SYSTEM DESCRIPTION}

A CPM modulator is a device with memory that generates continuous-phase, constant-envelope modulated waveforms

$$
x(t)=\sqrt{\frac{2 E_{s}}{T}} e^{j \psi(t)}
$$

whose phase

$$
\psi(t)=2 \pi h \sum_{n=-\infty}^{\infty} a_{n} q(t-n T)
$$

depends on the input information symbols $a_{n} \in$ $\{ \pm 1, \pm 3, \ldots, \pm(M-1)\}$, where $M=2^{m}$ is the size of the input alphabet. Here, $T$ is the symbol interval, $E_{s}$ is the energy per symbol, $h=Q / P$ is the modulation index $(Q$ and $P$ are relatively prime integers), and $q(t)$ is the phase pulse, a continuous function with the following properties

$$
q(t)= \begin{cases}0 & t \leq 0 \\ \frac{1}{2} & t \geq L T\end{cases}
$$

The phase pulse is usually defined as the integral of a frequency pulse $s(t)$

$$
q(t)=\int_{-\infty}^{t} s(\tau) d \tau
$$

A CPM scheme is then defined by specifying its parameters $M, h, L$ and the frequency pulse $s(t)$.

In this paper, we will consider rectangular (REC) and raisedcosine (RC) frequency pulses. The REC pulse is defined as

$$
s_{\mathrm{REC}}(t)=\frac{1}{2 L T}[u(t)-u(t-L T)]
$$

and the RC pulse is defined as

$$
s_{\mathrm{RC}}(t)=\frac{\pi}{4 L T}[u(t)-u(t-L T)]\left[1-\cos \left(\frac{2 \pi t}{L T}\right)\right]
$$

where $u(t)$ is the unitary step function.

According to the well known Rimoldi decomposition [2], the modulator can be represented as the cascade of a continuous phase encoder (CPE) and a memoryless modulator $(\mathrm{MM})$ as in Fig. 1. The CPE, in general, is a time-invariant convolutional encoder operating on a ring of integers.

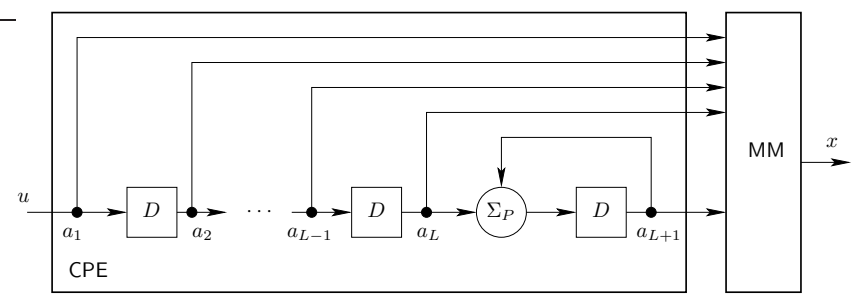

Fig. 1. Rimoldi decomposition of the CPM encoder. The block labelled $\Sigma_{P}$ is a modulo- $P$ adder.

The serial structure of the Rimoldi decomposition has been exploited by adding an outer convolutional encoder connected to the CPE trough an interleaver, so as to form a serially concatenated convolutional encoder (SCCC) with interleaver [17] (see Fig. 2). The iterative receiver performs decoding iterations between the inner SISO decoder, which operates on the CPE trellis, and the outer SISO decoder, which operates on the outer convolutional code trellis. This way, very good performance can be achieved, to be compared with the capacity of the CPM scheme. In the following, we will denote this scheme as serially concatenated CPM (SC-CPM) [4].

In this paper, we also consider a pragmatic approach, which results in a coded modulation scheme equivalent to a bitinterleaved coded modulation (BICM, see Fig. 3). It consists in

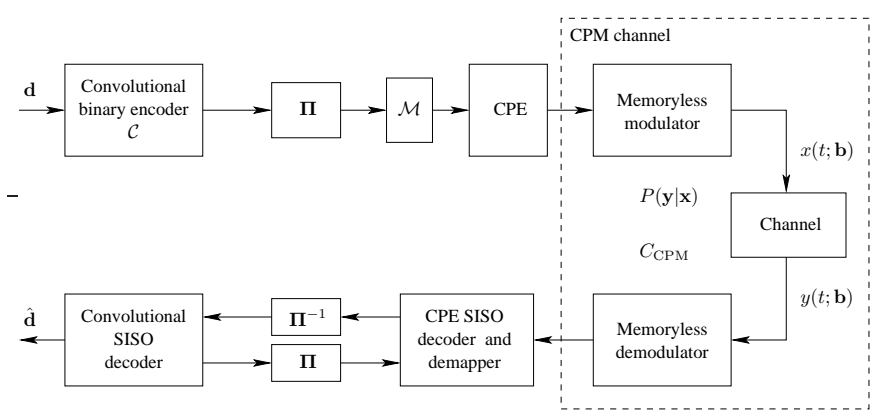

Fig. 2. Block diagram of a serially concatenated CPM co-decoder. The block labeled $\mathcal{M}$ maps blocks of $m$ bits to $M$-ary CPM symbols. 


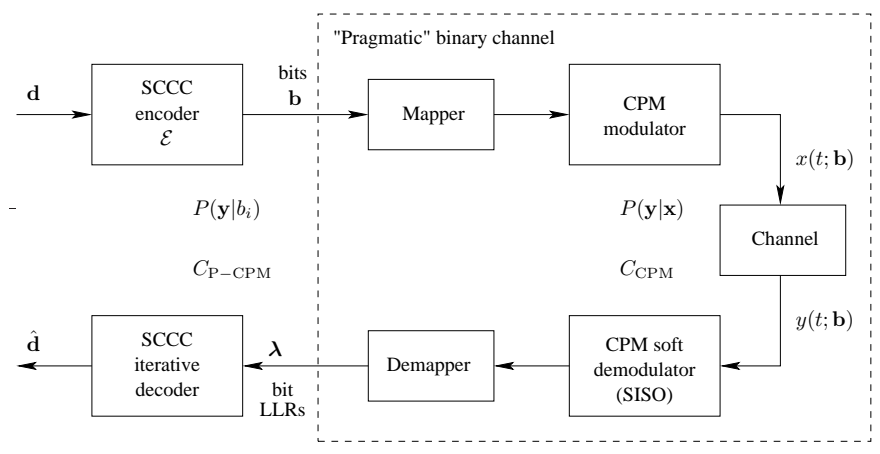

Fig. 3. Block diagram of an encoded CPM mo-demodulator used with a pragmatic approach.

cascading a powerful variable-rate binary encoder (a seriallyconcatenated convolutional code (SCCC) with interleaver in this paper) with the CPM modulator. The CPM modulator input is connected to the output of the channel encoder that computes the binary sequence $\mathbf{b}=\left(b_{i}, i \in \mathbb{Z}\right)$ from the binary information sequence $d$. Then,the coded binary sequence is mapped to the CPM modulator that generates a corresponding CPM signal sequence $x(t ; \mathbf{b})$. We will call this scheme pragmatic CPM (P-CPM).

The channel is an additive white Gaussian noise (AWGN) channel, whose output signal is $y(t ; \mathbf{b})=x(t ; \mathbf{b})+n(t)$, where $n(t)$ is a zero-mean white Gaussian process with two-sided power spectral density $N_{0} / 2$. At the receiver, the CPM soft demodulator and demapper provide the sequence $\boldsymbol{\lambda}=\left(\lambda_{i}, i \in\right.$ $\mathbb{Z}$ ) of log-likelihood ratios on the information bit sequence to the outer decoder

$$
\lambda_{i}=\log \frac{P\left(b_{i}=1 \mid y(t ; \mathbf{b})\right)}{P\left(b_{i}=0 \mid y(t ; \mathbf{b})\right)}
$$

Finally, $\boldsymbol{\lambda}$ is used by the outer iterative decoder to compute the information sequence estimate $\hat{\mathbf{d}}$.

The main difference between P-CPM and SC-CPM is that no iterations are performed between the inner CPM demodulator and the outer turbo decoder. Thus, it keeps the nice features of all pragmatic approaches, which merge independent binary codes with higher order modulations without requiring joint optimization.

Our goal is to design coded modulation schemes based on CPM capable of transmitting information at rates close to the CPM channel capacity ${ }^{1}$ Such goal will be pursued by analyzing the P-CPM and SC-CPM schemes and choosing the best trade-off between the following characteristics:

- Spectral efficiency.

- Energy efficiency.

- Decoding complexity.

Spectral efficiency and energy efficiency are two wellknown concepts. The decoding complexity is defined here as the overall number of trellis edges per information bit visited by the decoding algorithm. This definition depends on the type

\footnotetext{
${ }^{1}$ Hereafter, with CPM channel capacity we mean constrained capacity with uniform input distribution.
}

of coded modulation being used (i.e., P-CPM or SC-CPM). For the P-CPM scheme, we have

$$
\mathcal{Y}_{\mathrm{P}-\mathrm{CPM}}=\mathcal{Y}_{\mathrm{SCCC}}+\mathcal{Y}_{\mathrm{CPM}}
$$

where

$$
\mathcal{Y}_{\mathrm{CPM}}=M N_{s, C P E} \frac{N_{o}}{m K_{o}}
$$

and $N_{o}$ and $K_{o}$ are, respectively, the outer code word length and information word length, and $N_{s, C P E}=P M^{L-1}$ is the number of states of the CPE. The complexity of the SCCC binary decoder is $\mathcal{Y}_{\mathrm{SCCC}}$ and will be defined later according to the chosen code (see (VII.1).

For the SC-CPM scheme, we adopt the following definition, which holds for binary convolutional codes with rate $\frac{K_{o}}{N_{o}}$ obtained by puncturing a rate $1 / 2$ mother code:

$$
\mathcal{Y}_{\mathrm{SC}-\mathrm{CPM}}=N_{i t}\left(2 N_{s o}+\mathcal{Y}_{\mathrm{CPM}}\right)
$$

where $N_{i t}$ is the number of decoding iterations between the outer SISO and the inner CPE SISO, and $N_{s o}$ is the number of states of the outer convolutional encoder.

In the following, we will optimize the CPM schemes to be embedded into both SC-CPM and P-CPM starting from their capacity evaluation.

\section{CPM CAPACITY COMPUTATION}

With reference to Figures 2 and 3, CPM signals are infinitelength waveforms. Consider then a finite observation window $[-N T, N T]$ of $2 N+1$ symbol intervals, and define the channel mutual information over it:

$$
I(\mathbf{X} ; \mathbf{Y})=E_{\mathbf{x}, \mathbf{y}}\left\{\log _{2} \frac{p_{\mathbf{Y} \mid \mathbf{X}}(\mathbf{y} \mid \mathbf{x})}{p_{\mathbf{Y}}(\mathbf{y})}\right\}
$$

where $\mathbf{X}$ is a vector of samples of $x(t)$ that form a sufficient statistic of $x(t)$ in the interval $[-N T, N T]$ and $\mathbf{Y}$ is the correspondent vector of channel outputs. The set of all possible values for $\mathbf{X}$ will be denoted $\mathcal{X}$ throughout the paper. The CPM capacity can be defined through the limit

$$
C_{\mathrm{CPM}}=\lim _{N \rightarrow \infty} \frac{1}{(2 N+1) T} I(\mathbf{X} ; \mathbf{Y})[\mathrm{bits} / \mathrm{s}]
$$

Using the definition of mutual information $I$ and of the log-likelihood ratio (LLR)

$$
\lambda(\mathbf{u}, \mathbf{y}) \triangleq \log _{2}\left(p_{\mathbf{Y} \mid \mathbf{X}}(\mathbf{y} \mid \mathbf{u})\right)-\log _{2}\left(p_{\mathbf{Y} \mid \mathbf{X}}\left(\mathbf{y} \mid \mathbf{u}_{\mathrm{ref}}\right)\right)
$$

as well as the $\max ^{*}$ operator [17]

$$
\max ^{*}(a, b) \triangleq \log _{2}\left(2^{a}+2^{b}\right)
$$

we can transform (III.1) into

$$
C_{\mathrm{CPM}}=m-\lim _{N \rightarrow \infty} \frac{1}{(2 N+1) T} E_{\mathbf{x}, \mathbf{y}}\left\{\max _{\mathbf{u} \in \mathcal{X}}^{*} \lambda(\mathbf{u}, \mathbf{y})-\lambda(\mathbf{x}, \mathbf{y})\right\} .
$$

where $m$ is the number of bits per CPM symbol.

It has been observed in [7] that the first term inside the average is a by-product of the SISO algorithm and corresponds 
to the $\max ^{*}$ of the forward path metrics $\alpha$ at step $N$ in the CPE decoder

$$
\max _{\mathbf{u} \in \mathcal{X}}^{*} \lambda(\mathbf{u}, \mathbf{y})=\max _{s}{ }^{*} \alpha_{N}(s),
$$

with the following initialization

$$
\alpha_{-N}(s)= \begin{cases}0, & s=0 \\ -\infty, & s \neq 0\end{cases}
$$

where $s$ runs in the set of trellis states of the CPE. Moreover, since the channel is memoryless, the second term is obtained by summing the LLRs of the transmitted waveforms:

$$
\lambda(\mathbf{x}, \mathbf{y})=\sum_{i=-N}^{N} \lambda\left(x_{i}, y_{i}\right)
$$

Finally, invoking the ergodic properties of the system, the ensemble average can be removed leading to:

$C_{\mathrm{CPM}}=m-\lim _{N \rightarrow \infty} \frac{1}{2 N+1}\left(\max _{\mathbf{u}} \in \mathcal{X}^{*} \lambda(\mathbf{u}, \mathbf{y})-\lambda(\mathbf{x}, \mathbf{y})\right)$.

Thus, the CPM capacity can be estimated through a MonteCarlo simulation of the three internal blocks of Fig. 3 , i.e., the CPM modulator, the channel, and the CPM soft demodulator, followed by a time average.

\section{OPTIMIZATION PROCEDURE}

We define the complexity of a CPM scheme as the number of edges per CPM input bit' $\mathcal{Y}=P 2^{m L} / m$, where the parameters have been defined in Section II. Our aim is to maximize the CPM capacity measured in bits/s/Hertz versus the signal-to-noise ratio for a given complexity. The optimization algorithm modifies the CPM parameters $(m, P$, and $L)$ yielding the given complexity, and computes the CPM capacity expressed in bits per CPM waveform for a given signal-tonoise ratio according to the algorithm described in Sec. III Then, based on a bandwidth definition of the CPM scheme, it evaluates the rate and chooses the best scheme for all signalto-noise ratios of interest. In the following, we describe the optimization algorithm step by step:

1) Define the bandwidth $B$ of the CPM system that contains a given percentage of the total signal power.

2) Define the CPM symbol rate as $R_{s} \triangleq 1 / T$.

3) The symbol signal-to-noise ratio $E_{s} / N_{0}$ is given by

$$
\frac{E_{s}}{N_{0}}=\frac{1}{R_{s}} \frac{P_{T}}{N_{0}}
$$

where $P_{T}$ is the transmitted power.

4) Compute the capacity (in bits/symbol) of each CPM scheme using the method described in Section III as a function of the symbol SNR $C_{\mathrm{CPM}}=C_{\mathrm{CPM}}\left(E_{s} / N_{0}\right)$.

5) Evaluate the CPM capacity as

$$
C=R_{s} C_{\mathrm{CPM}}\left(\frac{1}{R_{s}} \frac{P_{T}}{N_{0}}\right) \quad[\mathrm{bits} / \mathrm{s} / \mathrm{Hz}]
$$

${ }^{2}$ Please note that the definition of $\mathcal{Y}$ differs from $\mathcal{Y}_{\mathrm{CPM}}$ in that the former is the number of CPE trellis edges per CPM input bit, while the latter is the number of CPE trellis edges per bit at the channel encoder input.
6) All CPM schemes with a given complexity $\mathcal{Y}$ are compared with respect to $C$ for each value of $\frac{P_{T}}{N_{0}}$ and the best is chosen.

7) Finally, to obtain the normalized plot that uses the bit SNR $E_{b} / N_{0}$ on the abscissa we use the relationship

$$
\frac{E_{b}}{N_{0}}=\frac{1}{C_{\mathrm{CPM}}} \frac{E_{s}}{N_{0}}=\frac{R_{s}}{C} \frac{E_{s}}{N_{0}}=\frac{1}{C} \frac{P_{T}}{N_{0}} .
$$

In Fig. 4 and Fig. 5 we show the capacity versus $E_{b} / N_{0}$ for the best CPM schemes with REC and RC frequency pulses and complexity ranging from 8 to 512 . The bandwidth is defined as the one including $99 \%$ of the total signal power. For comparison purposes, in the figure we have also plotted the capacity of QPSK and 8PSK modulations with a square root raised cosine shaping filter with roll-off 0.25 , and the unconstrained capacity of the AWGN channel.

In Table II and Table II we list for each SNR $\left(E_{s} / N_{0}\right)$ and complexity from 8 to 512 the CPM schemes with REC and $\mathrm{RC}$ frequency pulses achieving the highest capacity for a given signal-to-noise ratio. Each scheme is characterized by the parameters $E_{b} / N_{0}$ as defined in $\overline{I V .2}$, the capacity $C$ as defined in (IV.1), the CPM parameters $m, L, P$, and finally the symbol rate $R_{s}$.

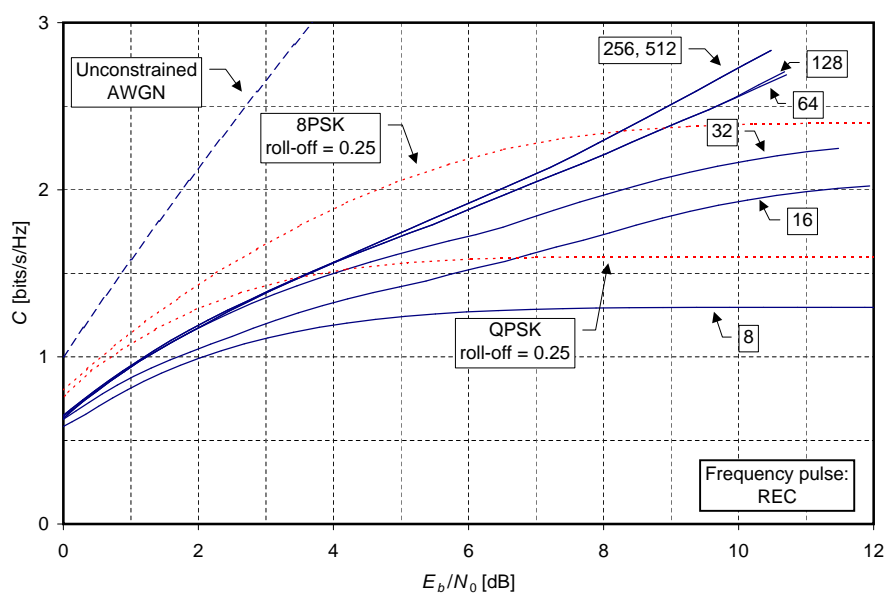

Fig. 4. Capacity versus $E_{b} / N_{0}$ of the best CPM schemes with rectangular frequency pulse and variable complexity. The bandwidth is defined at $99 \%$ of the total power.

\section{PRAGMATIC CAPACITY OF A TCM SCHEME}

With reference to Fig. 3, let the binary sequence $\mathbf{b}$ be formed by vectors $\mathbf{B}_{n}=\left(B_{n, 1}, \ldots, B_{n, m}\right)$ of the $m$ bits entering the CPM modulator at the $n$-th trellis step. As previously, we consider a length- $(2 N+1)$ input sequence of $\mathbf{B}_{n}$.

The scheme of Fig. 3 can be characterized by a pragmatic capacity $C_{\mathrm{P}-\mathrm{CPM}}$, defined by:

$$
C_{\mathrm{P}-\mathrm{CPM}} \triangleq \lim _{N \rightarrow \infty} \frac{1}{2 N+1} \sum_{n=-N}^{N} \sum_{i=1}^{m} I\left(B_{n, i} ; \mathbf{Y}\right),
$$

which depends on the mapping between input binary sequences and CPM waveforms.

In order to optimize the system performance, it is crucial to search for the optimal mapping, i.e., the one that maximizes the pragmatic capacity of a given CPM scheme. To this 


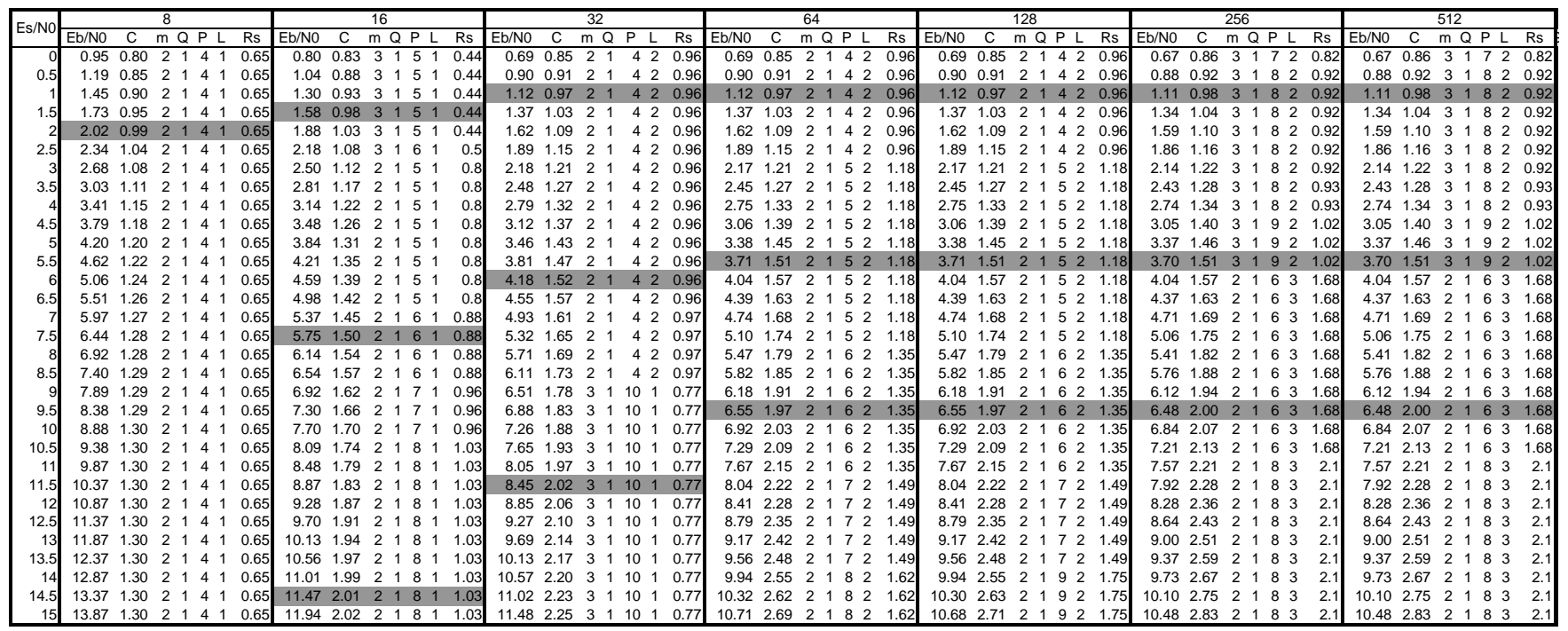

TABLE I

TABLE OF THE CPM SCHEMES With BEST $C$ AND RECTANGULAR FREQUENCY PULSE AT 99\% BANDWIDTH. THE COMPLEXITY RANGE IS 8 TO 512 . THE HIGHLIGHTED ENTRIES CORRESPOND TO THE SELECTED CPM SCHEMES FOR TARGET CAPACITIES OF 1, 1.5 AND 2 BITS/S/Hz.

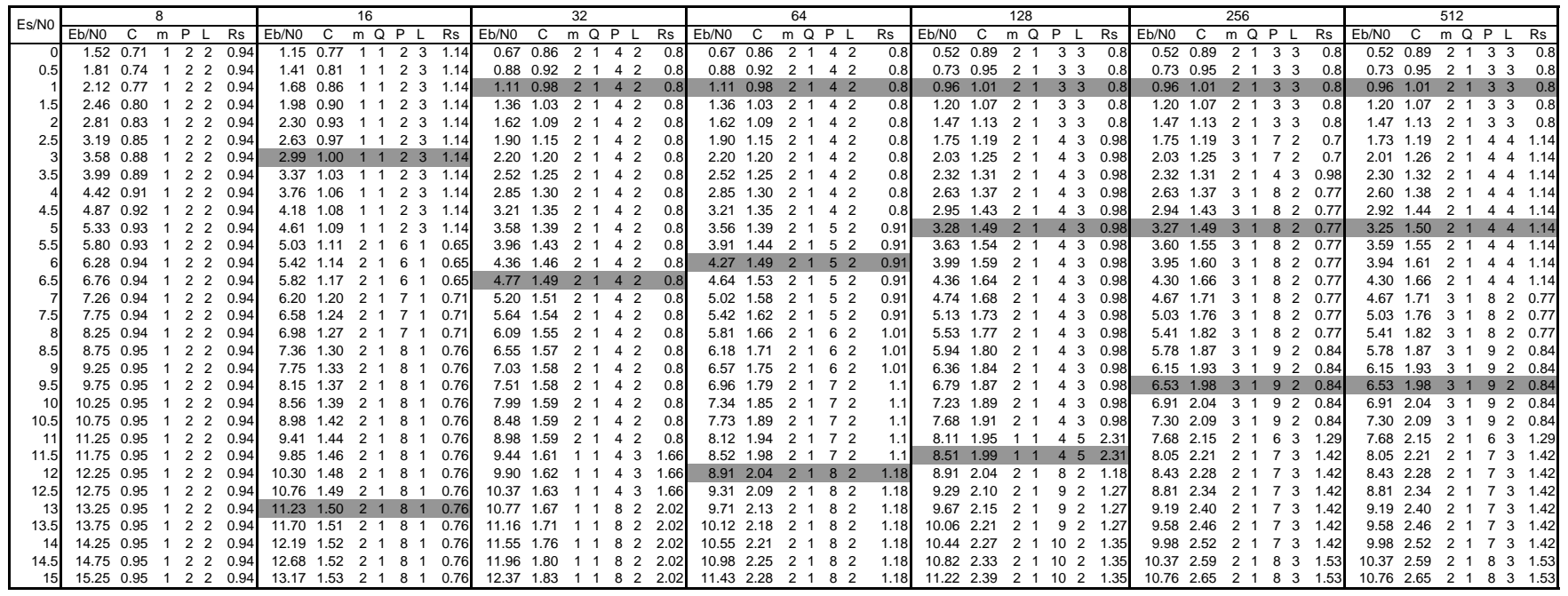

TABLE II

TABLE OF THE CPM SCHEMES WITH BEST $C$ AND RAISED-COSINE FREQUENCY PULSE AT 99\% BANDWIDTH. THE COMPLEXITY RANGE IS 8 TO 512. THE HIGHLIGHTED ENTRIES CORRESPOND TO THE SELECTED CPM SCHEMES FOR TARGET CAPACITIES OF 1, 1.5 AND 2 BITS/S/Hz.

purpose, we will derive in the following a lower bound to the pragmatic capacity, which makes explicit the dependence on the mapping. Before delving deeper into the analysis, though, we show in Fig. 6 a comparison between the optimized PCPM capacity, obtained through the algorithm derived in the following, and that obtained by a straightforward application of the mapping induced by the Rimoldi decomposition. The curves show that the pragmatic capacity of the optimized CPE is significantly improved with respect to the non optimized CPE. For the non optimized CPE, the gap between the CPM capacity and the pragmatic capacity is roughly $1.5 \mathrm{~dB}$, while the pragmatic capacity of the optimized CPE is very close to the CPM capacity. The obtained gain is roughly $1.4 \mathrm{~dB}$.
Since

$$
I\left(B_{n, i} ; \mathbf{Y}\right)=1-H\left(B_{n, i} \mid \mathbf{Y}\right),
$$

the optimal mapping is the one that yields a minimum of $\sum_{n} \sum_{i} H\left(B_{n, i} \mid \mathbf{Y}\right)$. Define $\mathcal{X}_{n, i}(b), b=0,1$, as the set of CPM waveforms whose mapping satisfies $B_{n, i}=b$. Obviously, $\mathcal{X}=\mathcal{X}_{n, i}(0) \cup \mathcal{X}_{n, i}(1)$. We have:

$$
H\left(B_{n, i} \mid \mathbf{Y}\right)=\int H\left(B_{n, i} \mid \mathbf{Y}=\mathbf{y}\right) p_{\mathbf{Y}}(\mathbf{y}) d \mathbf{y} .
$$

Now, $H\left(B_{n, i} \mid \mathbf{Y}=\mathbf{y}\right)$ is given explicitly by:

$$
H\left(B_{n, i} \mid \mathbf{Y}=\mathbf{y}\right)=H\left(\frac{\sum_{\mathbf{u} \in \mathcal{X}_{n, i}(0)} p_{\mathbf{Y} \mid \mathbf{X}}(\mathbf{y} \mid \mathbf{u})}{\sum_{\mathbf{u} \in \mathcal{X}} p_{\mathbf{Y} \mid \mathbf{X}}(\mathbf{y} \mid \mathbf{u})}\right),
$$




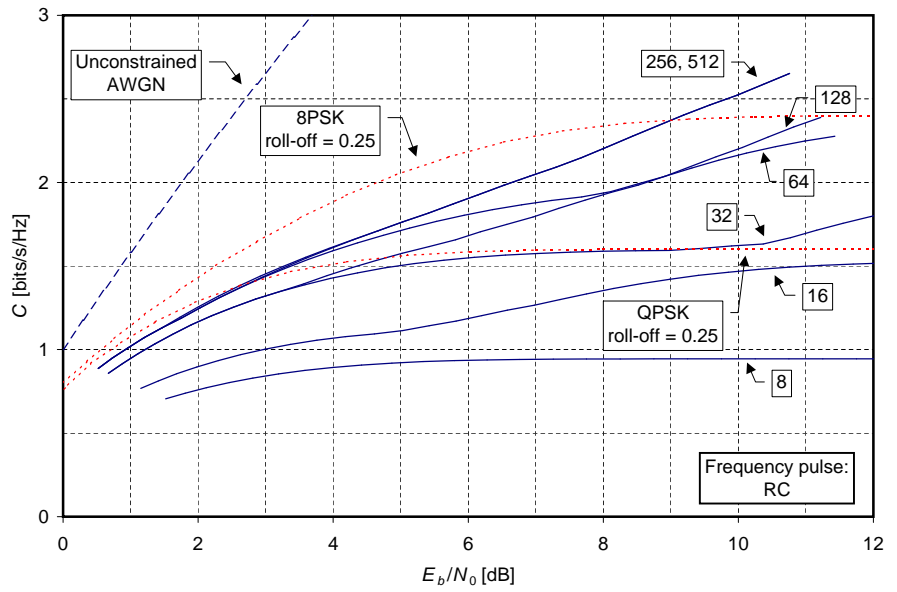

Fig. 5. Capacity versus $E_{b} / N_{0}$ of the best CPM schemes with raised-cosine frequency pulse and variable complexity. The bandwidth is defined at $99 \%$ of the total power.

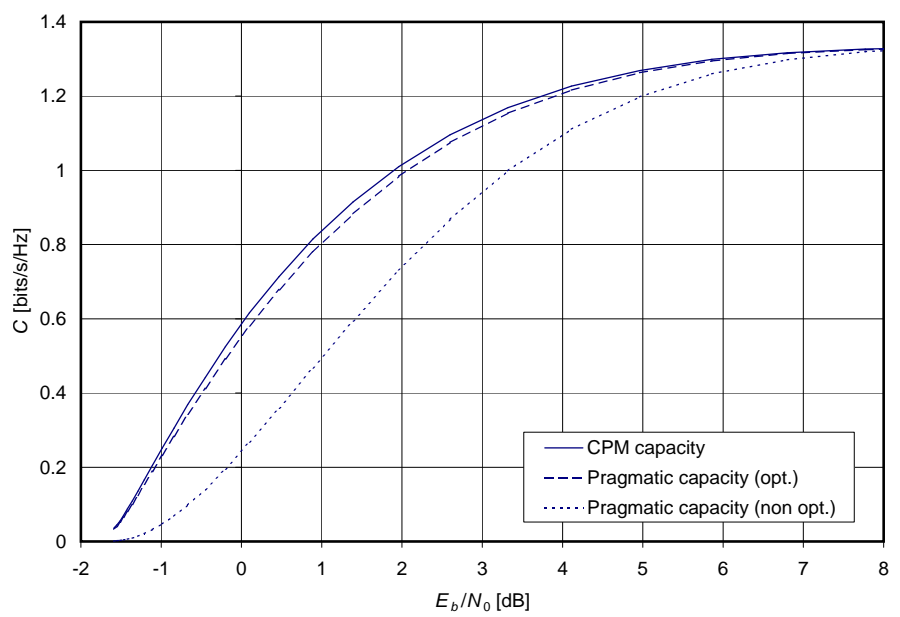

Fig. 6. CPM capacity and pragmatic capacities of the binary REC scheme with $h=1 / 2$ and $L=3$.

where the binary entropy function $H(p)=-p \log p-(1-$ p) $\log (1-p)$ has been used in the RHS of the above equation.

To find a viable path to mapping optimization, we approximate the distribution of $\mathbf{Y}$ with its value for infinite SNR, by making in (V.3) the following substitution:

$$
p_{\mathbf{Y}}(\mathbf{y}) \approx \frac{1}{|\mathcal{X}|} \sum_{\mathbf{x} \in \mathcal{X}} \delta(\mathbf{x}-\mathbf{y}),
$$

where $|\mathcal{X}|$ is the size of $\mathcal{X}$ and $\delta(\cdot)$ is Dirac's delta. The above approximation makes sense because the optimal mapping for large SNR should reasonably be the same as for infinite SNR.

Substituting (V.5) into V.3, we obtain:

$$
\begin{aligned}
H\left(B_{n, i} \mid \mathbf{Y}\right) \approx & \frac{1}{2} \sum_{b=0,1} \frac{2}{|\mathcal{X}|} \times \\
& \sum_{\mathbf{x} \in \mathcal{X}_{n, i}(b)} H\left(\frac{\sum_{\mathbf{u} \in \mathcal{X}_{n, i}(0)} p_{\mathbf{Y} \mid \mathbf{X}}(\mathbf{x} \mid \mathbf{u})}{\sum_{\mathbf{u} \in \mathcal{X}} p_{\mathbf{Y} \mid \mathbf{X}}(\mathbf{x} \mid \mathbf{u})}\right),
\end{aligned}
$$

where the sum w.r.t $\mathrm{x}$ over $\mathcal{X}$ has been split into separate sums over $\mathcal{X}_{n, i}(0)$ and $\mathcal{X}_{n, i}(1)$.
By Jensen's inequality and the concavity of the entropy function, we obtain the upper bound:

$H\left(B_{n, i} \mid \mathbf{Y}\right) \leq \frac{1}{2} \sum_{b=0,1} H\left(\frac{2}{|\mathcal{X}|} \sum_{\mathbf{x} \in \mathcal{X}_{n, i}(b)} \frac{\sum_{\mathbf{u} \in \mathcal{X}_{n, i}(0)} p_{\mathbf{Y} \mid \mathbf{X}}(\mathbf{x} \mid \mathbf{u})}{\sum_{\mathbf{u} \in \mathcal{X}} p_{\mathbf{Y} \mid \mathbf{X}}(\mathbf{x} \mid \mathbf{u})}\right)$.

Owing to the ergodic property of the system, it can be shown that $\sum_{\mathbf{u} \in \mathcal{X}} p_{\mathbf{Y} \mid \mathbf{X}}(\mathbf{x} \mid \mathbf{u})$ is equal to a constant for almost every transmitted $\mathbf{x}$. Thus:

$H\left(B_{n, i} \mid \mathbf{Y}\right) \leq \frac{1}{2} \sum_{b=0,1} H\left(\frac{2 \sum_{\mathbf{x} \in \mathcal{X}_{n, i}(b)} \sum_{\mathbf{u} \in \mathcal{X}_{n, i}(0)} p_{\mathbf{Y} \mid \mathbf{X}}(\mathbf{x} \mid \mathbf{u})}{\sum_{\mathbf{x} \in \mathcal{X}} \sum_{\mathbf{u} \in \mathcal{X}} p_{\mathbf{Y} \mid \mathbf{X}}(\mathbf{x} \mid \mathbf{u})}\right)$.

Finally, notice that, since $p_{\mathbf{Y} \mid \mathbf{X}}(\mathbf{x} \mid \mathbf{u})=p_{\mathbf{Y} \mid \mathbf{X}}(\mathbf{u} \mid \mathbf{x})$ for the AWGN channel, the two entropies in the RHS of $(\mathrm{V} .8)$ have the same arguments, and thus are equal:

$$
H\left(B_{n, i} \mid \mathbf{Y}\right) \leq H\left(\frac{2 \sum_{\mathbf{x} \in \mathcal{X}_{n, i}(0)} \sum_{\mathbf{u} \in \mathcal{X}_{n, i}(0)} p_{\mathbf{Y} \mid \mathbf{X}}(\mathbf{x} \mid \mathbf{u})}{\sum_{\mathbf{x} \in \mathcal{X}} \sum_{\mathbf{u} \in \mathcal{X}} p_{\mathbf{Y} \mid \mathbf{X}}(\mathbf{x} \mid \mathbf{u})}\right)
$$

or, in a more explicit form:

$$
\begin{array}{r}
H\left(B_{n, i} \mid \mathbf{Y}\right) \leq-\frac{2 \sum_{\mathbf{x} \in \mathcal{X}_{n, i}(0)} \sum_{\mathbf{u} \in \mathcal{X}_{n, i}(0)} p_{\mathbf{Y} \mid \mathbf{X}}(\mathbf{x} \mid \mathbf{u})}{\sum_{\mathbf{x} \in \mathcal{X}} \sum_{\mathbf{u} \in \mathcal{X}} p_{\mathbf{Y} \mid \mathbf{X}}(\mathbf{x} \mid \mathbf{u})} \times \\
\log \frac{2 \sum_{\mathbf{x} \in \mathcal{X}_{n, i}(0)} \sum_{\mathbf{u} \in \mathcal{X}_{n, i}(0)} p_{\mathbf{Y} \mid \mathbf{X}}(\mathbf{x} \mid \mathbf{u})}{\sum_{\mathbf{x} \in \mathcal{X}} \sum_{\mathbf{u} \in \mathcal{X}} p_{\mathbf{Y} \mid \mathbf{X}}(\mathbf{x} \mid \mathbf{u})} \\
-\frac{2 \sum_{\mathbf{x} \in \mathcal{X}_{n, i}(0)} \sum_{\mathbf{u} \in \mathcal{X}_{n, i}(1)} p_{\mathbf{Y} \mid \mathbf{X}}(\mathbf{x} \mid \mathbf{u})}{\sum_{\mathbf{x} \in \mathcal{X}} \sum_{\mathbf{u} \in \mathcal{X}} p_{\mathbf{Y} \mid \mathbf{X}}(\mathbf{x} \mid \mathbf{u})} \times \\
\log \frac{2 \sum_{\mathbf{x} \in \mathcal{X}_{n, i}(0)} \sum_{\mathbf{u} \in \mathcal{X}_{n, i}(1)} p_{\mathbf{Y} \mid \mathbf{X}}(\mathbf{x} \mid \mathbf{u})}{\sum_{\mathbf{x} \in \mathcal{X}} \sum_{\mathbf{u} \in \mathcal{X}} p_{\mathbf{Y} \mid \mathbf{X}}(\mathbf{x} \mid \mathbf{u})}
\end{array}
$$

The above equation, substituted in $(\mathrm{V} .2$ and, then, back in (V.1), gives the lower bound on the pragmatic capacity that is the starting point for our optimization.

\section{A. Mapping optimization}

The problem of mapping optimization has been reduced to the minimization of the upper bound in (V.9) (or $(\mathrm{V})$ ). A binary entropy function is minimized if its argument distribution is made as unbalanced as possible. For large SNR, $\sum_{\mathbf{x} \in \mathcal{X}_{n, i}(0)} \sum_{\mathbf{u} \in \mathcal{X}_{n, i}(0)} p_{\mathbf{Y} \mid \mathbf{X}}(\mathbf{x}, \mathbf{u})$ will be the dominant term in (V.9), because it contains the term with $\mathbf{x}=\mathbf{u}$; thus, it should be made as close to 1 as possible. Conversely, the complementary term $\sum_{\mathbf{x} \in \mathcal{X}_{n, i}(0)} \sum_{\mathbf{u} \in \mathcal{X}_{n, i}(1)} p_{\mathbf{Y} \mid \mathbf{X}}(\mathbf{x}, \mathbf{u})$, should be made as close to 0 as possible, i.e., it should be minimized. We have come up with the following design rule for the optimal mapping:

$$
\mathcal{X}_{n, i}(0)=\arg \min _{\substack{\mathcal{X}^{\prime} \subset \mathcal{X} \\\left|\mathcal{X}^{\prime}\right|=|\mathcal{X}| / 2}} \sum_{\mathbf{x} \in \mathcal{X}^{\prime}} \sum_{\mathbf{u} \notin \mathcal{X}^{\prime}} p_{\mathbf{Y} \mid \mathbf{X}}(\mathbf{x}, \mathbf{u}) .
$$

For practical reasons, the mapping is generated by an $m$ bit labelling of the trellis edges. Let $\mathcal{T}_{n, i}(0)\left(\mathcal{T}_{n, i}(1)\right)$ be the subset of trellis edges at time $n$ whose label has a 0 (1) in the $i$-th position. Then, $\mathcal{X}_{n, i}(0)$ is constituted by all CPM waveforms whose trellis paths pass through an edge belonging to $\mathcal{T}_{n, i}(0)$, and the design rule in $\mathrm{V} .10$ can be restated in terms of $\mathcal{T}_{n, i}(0)$. 
We impose the condition that the trellis labelling must be right-resolving, i.e., edges leaving the same trellis state have different binary labels. This condition does not force a trellis labelling that varies with $n$. Moreover, the design criterion in V.10 is also independent of $n$. From these facts, we deduce that the optimal mapping is generated by a time-invariant trellis labelling. Thus $\mathcal{T}_{n, i}(0)=\mathcal{T}_{i}(0)$, for every $n$. Instead, the condition of having a right-resolving trellis implies that $\mathcal{T}_{1}(0), \ldots, \mathcal{T}_{m}(0)$ cannot be chosen independently, for $m>1$.

In practice, we approximate the design rule in (V.10) with the following suboptimal rule, which derives from the assumption that, for large SNR, there is one dominant term in the sum of (V.10):

$$
\mathcal{X}_{n, i}(0)=\arg \min _{\mathcal{X}^{\prime} \subset \mathcal{X}}\left\{\max _{\mathbf{x} \in \mathcal{X}^{\prime}} \max _{\mathbf{u} \notin \mathcal{X}^{\prime}} p_{\mathbf{Y} \mid \mathbf{X}}(\mathbf{x}, \mathbf{u})\right\} .
$$

Based on (V.11), our mapping optimization procedure consists then of the following steps:

- For every pair of trellis edges, we apply the BCJR algorithm to compute $\max _{\mathbf{u}} \max _{\mathbf{x}} p_{\mathbf{Y} \mid \mathbf{X}}(\mathbf{x}, \mathbf{u})$ over all pairs of paths passing through the given pair of edges at time zero. To do this in general, we have to extend the trellis both at the left (negative time instants) and at the right (positive time instants) of the zeroth section. After a few trellis steps, the path metrics reach a steady-state value, so there is no need to proceed further.

- The previous step yields a metric for each pair of edges. Since we want to minimize the expression between braces in $(\mathrm{V} .11)$, we partition the edges into $M=2^{m}$ equal-size clusters, corresponding to the $M$ different $m$-bit labels, in such a way that pairs of edges with the highest metric are all clustered together. In doing this, we force pairs of edges with the same starting state into different clusters, to allow for a right-resolving labelling.

- We map the $M=2^{m}$ clusters to $m$-tuples of bits according to a Gray mapping. More precisely, we define the distance between two clusters as the minimum distance between CPM waveforms passing at time zero through edges belonging to the two clusters. Cluster pairs with the smallest distance will be associated to binary labels with Hamming distance 1.

The highest metric corresponds to edge pairs that belong to the same trellis section of a minimum-distance error event. It may be questionable whether it is possible to cluster all pairs of edges with that property, apart from those with the same starting state, and whether this gives a unique clustering. In the next subsection, we will completely answer these questions for $M=2$, and give the analytical expression of the optimal mapping, provided that some necessary conditions are met.

\section{B. The optimal mapping for $M=2$}

As described in the previous subsection, the algorithm for mapping optimization puts into the same cluster all edge pairs that belong to the same trellis section of a minimum-distance error event. For labelling purposes, we would like to obtain in this way exactly $M$ clusters, but this happens only at certain conditions. In this subsection, we derive such conditions for binary CPM schemes.
Let us consider a CPM scheme with $M=2$, impulse length $L$ and modulation index $h=Q / P$. Trellis edges will be denoted hereafter through the couple $(\alpha, \beta)$, where $\alpha$ is a length- $L$ binary vector, including the correlative state and the input symbol, and $\beta \in\{0, \ldots, P-1\}$ represents the phase state. Let $x_{1}(t)$ and $x_{2}(t)$ be two CPM waveforms, corresponding to input symbol sequences $\mathbf{a}_{1}$ and $\mathbf{a}_{2}$, respectively. It is well known that the Euclidean distance between these two CPM waveforms only depends on the difference sequence $\mathbf{b} \triangleq \mathbf{a}_{1}-\mathbf{a}_{2}$, with elements belonging to $\{-1,0,1\}$.

Now, consider a given difference sequence $\mathbf{b}$ and define $\mathbf{a}$ graph $\mathcal{G}(\mathbf{b})=(\mathcal{V}, \mathcal{E}(\mathbf{b}))$, where:

- the vertex set $\mathcal{V}$ is in one-to-one correspondence to the set of trellis edges for the considered CPM scheme.

- the edge set $\mathcal{E}(\mathbf{b})$ is constructed in the following way: two vertices are connected by an edge if and only if the corresponding trellis edges have different starting states and belong to the same trellis section of an error event generated by $\mathbf{b}$.

Let $\mathcal{C}_{0}, \ldots, \mathcal{C}_{\mathcal{N}(\mathbf{b})-1}$ be the $\mathcal{N}(\mathbf{b})$ connected components of the graph $\mathcal{G}(\mathbf{b})$. The following theorem gives the important properties of $\mathcal{C}_{0}, \ldots, \mathcal{C}_{\mathcal{N}(\mathbf{b})-1}$.

Theorem 5.1: Consider a CPM scheme with $M=2$, impulse length $L$ and modulation index $h=Q / P$. Given a difference sequence $\mathbf{b}$, with length $\Delta(\mathbf{b})$, the following facts about the graph $\mathcal{G}(\mathbf{b})$ defined above hold:

1) If $\Delta(\mathbf{b})=2$, then $\mathcal{N}(\mathbf{b})=P$, otherwise $\mathcal{N}(\mathbf{b})=1$.

2) If $\mathcal{N}(\mathbf{b})=P$, the connected component $\mathcal{C}_{i}, i=$ $0, \ldots, P-1$ is the subgraph induced by the subset of vertices:

$$
\mathcal{V}_{i}=\left\{\left(\alpha,\left(i-Q w_{H}(\alpha)\right)_{P}\right): \alpha \in\{0,1\}^{L}\right\},
$$

independently of b. All $\mathcal{C}_{i}$ 's have size $2^{L}$.

Proof: See Appendix I]

Suppose now that $\mathbf{b}$ gives the minimum Euclidean distance of the CPM scheme. We want to partition the edges into two clusters, namely, $\mathcal{T}(0)$ and $\mathcal{T}(1)$ (edges with a label 0 and edges with a label 1 , respectively). To meet all constraints given by the graph $\mathcal{G}(\mathbf{b})$, every subset $\mathcal{V}_{i}$ should be entirely contained into one of the two clusters. This is clearly not possible if $\Delta(\mathbf{b}) \neq 2$, because in that case $\mathcal{V}_{1}=\mathcal{V}$.

If $\Delta(\mathbf{b})=2$, instead, we can place exactly $P / 2$ subsets into $\mathcal{T}(0)$ and the other $P / 2$ into $\mathcal{T}(1)$. (Notice that the sizes of $\mathcal{T}(0)$ and of $\mathcal{T}(1)$ both must be equal to $P 2^{L-1}$.) This can be done only if $P$ is even, otherwise, one of the $\mathcal{V}_{i}$ 's must be split in two. Thus, we have proved the following proposition:

Proposition 5.1: Consider a CPM scheme with $M=2$, impulse length $L$ and modulation index $h=Q / P$. We can fully perform the algorithm of mapping optimization described in the previous section if and only if:

- Every difference sequence $\mathbf{b}$ giving the minimum Euclidean distance of the scheme has length two, and

- $P$ is even.

${ }^{3}$ The notation $l_{P}$ means " $l$ modulo $P$ ". 
If the conditions of Prop. 5.1 are satisfied, then, the optimal mapping is uniquely determined by the condition of rightresolving labelling. In fact, the two trellis edges leaving a given trellis state will belong to $\mathcal{V}_{i}$ and $\mathcal{V}_{(i+Q)_{P}}$, for some $i$. Thus, for the labelling to be right-resolving, it must be:

$$
\mathcal{T}(0)=\mathcal{V}_{0} \cup \mathcal{V}_{(2 Q)_{P}} \cup \cdots \cup \mathcal{V}_{((P-2) Q)_{P}}
$$

and

$$
\mathcal{T}(1)=\mathcal{V}_{Q_{P}} \cup \mathcal{V}_{(3 Q)_{P}} \cup \cdots \cup \mathcal{V}_{((P-1) Q)_{P}},
$$

or vice versa. In this way, all CPM waveform pairs at a minimum distance between each other will be associated to binary input sequence pairs with Hamming distance equal to 1 .

If the conditions of Prop. 5.1 are not satisfied, then the mapping optimization algorithm of the previous section cannot be fully performed. It means that some CPM waveform pairs with minimum distance will be associated to binary input sequence pairs with Hamming distance larger than 1. A reasonable approach in this case is to try to minimize the number of such CPM waveform pairs.

\section{The optimal mapping for $M>2$}

In non-binary CPM schemes, things become more involved. In general, we have not found necessary and sufficient conditions to fully perform the algorithm of mapping optimization.

Here, we give a generalization of Theorem 5.1, which however deals only with a subset of possible difference sequences.

Theorem 5.2: Consider a CPM scheme with $M>2$, impulse length $L$ and modulation index $h=Q / P$. Let $\mathbf{b}=$ $\left(b_{1}, \ldots, b_{\Delta(\mathbf{b})}\right)$ be a difference sequence with length $\Delta(\mathbf{b})$ and elements in $\{0, \pm 1, \ldots, \pm(M-1)\}$. Let $b_{\Delta(\mathbf{b})}= \pm 1$. The following facts about the graph $\mathcal{G}(\mathbf{b})$ defined above hold:

1) If $\Delta(\mathbf{b})=2$, then $\mathcal{N}(\mathbf{b})=P$, otherwise $\mathcal{N}(\mathbf{b})=1$.

2) If $\mathcal{N}(\mathbf{b})=P$, the connected component $\mathcal{C}_{p}, p=$ $0, \ldots, P-1$ is the subgraph induced by the subset of vertices

$$
\begin{aligned}
\mathcal{V}_{p}=\left\{\left(\alpha,\left(p-Q \sum_{l=1}^{L} a_{l}\right)_{P}\right):\right. \\
\left.\alpha=\left(a_{1}, \ldots, a_{L}\right) \in\{0, \ldots, M-1\}^{L}\right\},
\end{aligned}
$$

independently of b. All $\mathcal{C}_{p}$ 's have size $M^{L}$.

Proof: See Appendix II

Following the same arguments of the previous subsection, we thus obtain the following proposition, which gives only sufficient (not necessary) conditions to fully perform the mapping optimization algorithm.

Proposition 5.2: Consider a CPM scheme with $M>2$, impulse length $L$ and modulation index $h=Q / P$. We can fully perform the algorithm of mapping optimization described in the previous section if:

- Every difference sequence $\mathbf{b}$ giving the minimum Euclidean distance of the scheme has length two and $b_{2}=$ \pm 1 , and

- $P$ is a multiple of $M$.
If the conditions of Prop. 5.2 are satisfied, then, by constraining the resulting labelling to be right-resolving, we obtain the following $M$ clusters:

$$
\widetilde{\mathcal{T}}(i)=\mathcal{V}_{(i Q)_{P}} \cup \mathcal{V}_{((M+i) Q)_{P}} \cup \cdots \cup \mathcal{V}_{((P-M+i) Q)_{P}},
$$

for $i=0, \ldots, M-1$.

Finally, the clusters $\widetilde{\mathcal{T}}(i)$ 's are mapped to the $M$ binary labels according to Gray mapping.

In the cases that are not covered by the hypotheses of Theorem 5.2, the algorithm may or may not be fully performed depending on the scheme parameters and on the difference sequences $\mathbf{b}$ giving the minimum Euclidean distance. If the algorithm can be fully performed, in general, the $M$ clusters may not be as in (V.16.

\section{The optimized CPE}

Starting from $(\mathrm{V} .15)$, where we define

$$
a_{L+1}=\left(p-Q \sum_{l=1}^{L} a_{l}\right)_{P}
$$

we derive the input symbol $a_{1}$ to the CPE as the solution to the following equation

$$
\left(Q a_{1}\right)_{P}=\left(p-a_{L+1}-Q \sum_{l=2}^{L} a_{l}\right)_{P} .
$$

Since $P$ and $Q$ are relatively prime, such solution is unique. Moreover, from V.15 we have $a_{1} \in\{0, \ldots, M-1\}$ and, since also $M$ and $Q$ are relatively prime, $\left(Q a_{1}\right)_{M}$ entails a unique solution for $a_{1}$. As a result, we can write

$$
\left(Q a_{1}\right)_{M}=\left(p-a_{L+1}-Q \sum_{l=2}^{L} a_{l}\right)_{M} .
$$

Clustering as in $\mathrm{V} .16$ can be performed observing that the vertex subset indices in each cluster can be written as $p=((k M+i) Q)_{P}$. Inserting this in $\left.\mathrm{V} .17\right)$ and recalling that $P$ is a multiple of $M$ we obtain

$$
\begin{aligned}
\left(Q a_{1}\right)_{M} & =\left(i Q+k M Q-a_{L+1}-Q \sum_{l=2}^{L} a_{l}\right)_{M} \\
& =\left(i Q-a_{L+1}-Q \sum_{l=2}^{L} a_{l}\right)_{M} .
\end{aligned}
$$

where $i$ is the cluster label. Applying the distributive property of multiplication over addition in the ring $\mathbb{Z}_{M}$ we obtain

$$
a_{1}=\left(i-\left(a_{L+1} Q^{-1}\right)_{M}-\sum_{l=2}^{L} a_{l}\right)_{M} .
$$

Finally, the binary label $\mathbf{B}$ is obtained as $\mathbf{B}=G(i)$, where $G$ is the Gray function defined in [18, ch.20]. Fig. 7 shows the scheme of the optimized CPM encoder.

Adopting the optimized CPE, i.e., starting from the CPE structure of Fig. 7, a procedure similar to the one described in Sec. IV has been performed in order to select the CPM 


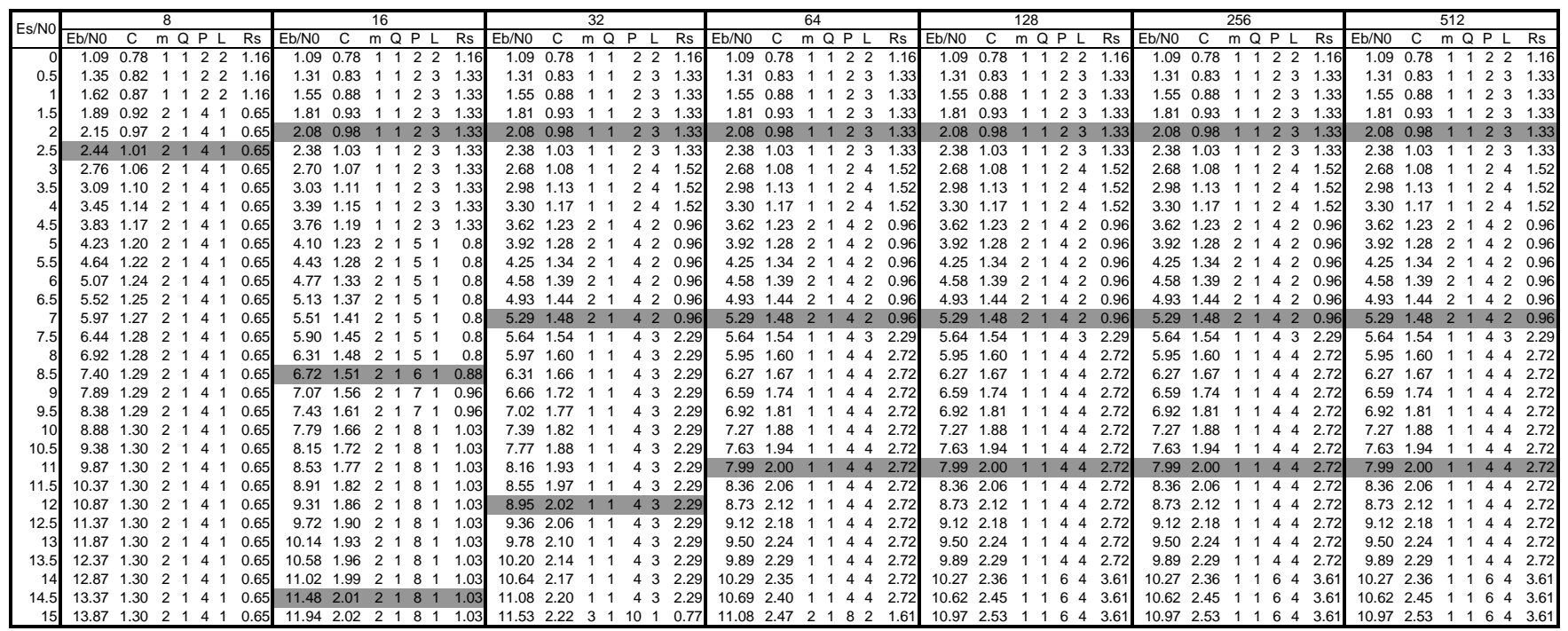

TABLE III

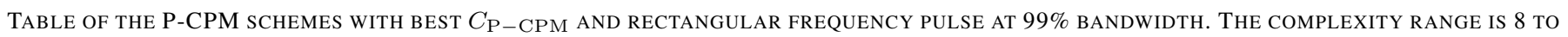
512. THE HIGHLIGHTED ENTRIES CORRESPOND TO THE SELECTED CPM SCHEMES FOR TARGET CAPACITIES OF $1,1.5$ AND 2 BITS/S/Hz.

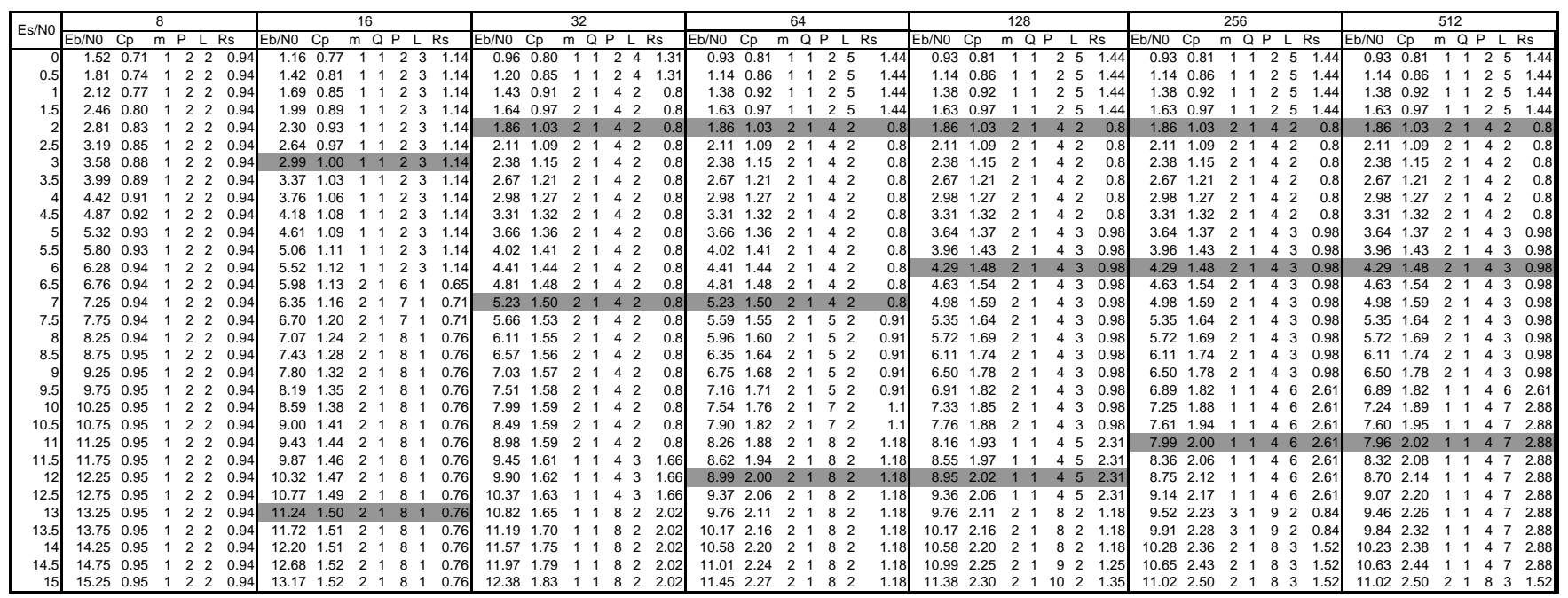

TABLE IV

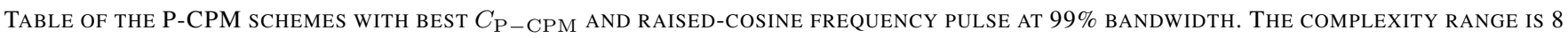
TO 512. THE HIGHLIGHTED ENTRIES CORRESPOND TO THE SELECTED CPM SCHEMES FOR TARGET CAPACITIES OF 1, 1.5 AND 2 BITS/S/HZ.

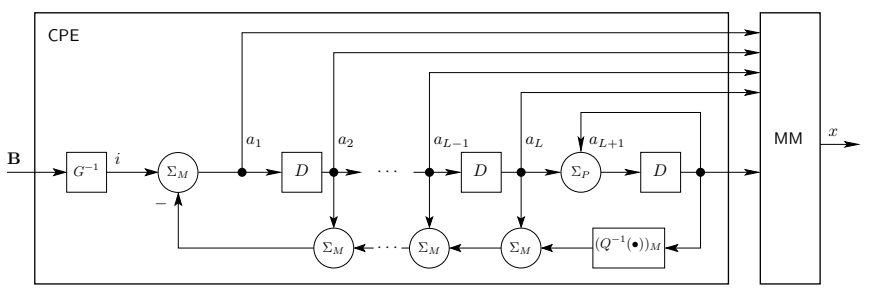

Fig. 7. The optimized CPM encoder. The block labelled $\Sigma_{P}$ (resp. $\Sigma_{M}$ ) is a modulo- $P$ (resp. modulo- $M$ ) adder. The block labelled $G^{-1}$ is the inverse of the Gray function defined in $[18, \mathrm{ch} .20]$.

schemes with best $C_{\mathrm{P}-\mathrm{CPM}}$. Results are shown in Tab. III and Tab. IV
A comparison of Tab. III with Tab. Iand Tab. IV with Tab. II highlights that, in some cases, the CPM schemes with best $C_{\mathrm{CPM}}$ feature the best $C_{\mathrm{P}-\mathrm{CPM}}$, as is the case, e.g., for REC pulses with complexity 64 and capacity $1.5 \mathrm{bits} / \mathrm{s} / \mathrm{Hz}$ and for RC pulses with complexity 64 and capacity $1.5 \mathrm{bits} / \mathrm{s} / \mathrm{Hz}$ and $2 \mathrm{bits} / \mathrm{s} / \mathrm{Hz}$. In some other cases, the selected schemes do not match.

We observed that, in many binary CPM schemes, the pragmatic capacity resulting from the CPE optimization approaches the CPM capacity closer than for non binary schemes. As a result, even when featuring a worse CPM capacity, these schemes may exhibit a larger pragmatic capacity. This is the case, e.g., of REC pulses with complexity 64 and capacity $1 \mathrm{bits} / \mathrm{s} / \mathrm{Hz}$, where the scheme with best $C_{\mathrm{CPM}}$ is 
quaternary and the scheme with best $C_{\mathrm{P}-\mathrm{CPM}}$ is binary.

The optimal mapping described above results in a CPM scheme with finite $d_{E 1}$, the minimum Euclidean distance at the CPM output corresponding to an input Hamming distance 1. To prove this, we use the polynomial notation with dummy variable $D$ for symbol sequences and we observe from Fig. 7 that

$$
a_{i+1}(D)=D a_{i}(D), \quad i=1, \ldots, L-1
$$

and

$$
a_{L+1}(D)=\left(a_{L}(D) \frac{D}{1-D}\right)_{P}
$$

If $Q=1$, from $(\mathrm{V} .20), \mathrm{V} .19$ ) and $(\mathrm{V} .18$, we obtain

$$
a_{1}(D)=(i(D)(1-D))_{M} .
$$

Therefore $a_{1}(D)$ can be obtained by feedforward encoding of $i(D)$. From V.19 it is straightforward to deduct that all $a_{i}(D), i=1, \ldots, L$ have the same property.

As for symbol $a_{L+1}(D)$, from (V.20) we obtain

$$
a_{L+1}(D)=\left(\frac{D^{L} a_{1}(D)}{1-D}\right)_{P}=\left(\frac{D^{L}(i(D)(1-D))_{M}}{1-D}\right)_{(\mathrm{V} .21)}
$$

If $M=z P, z \in \mathbb{N}$, this clearly proves that symbol $a_{L+1}(D)$ can be obtained by feedforward encoding $i(D)$. When $P=$ $w M, w \in \mathbb{N}$, it is possible to rewrite $\mathrm{V} .21$ as

$$
\begin{aligned}
a_{L+1}(D) & =\left(\frac{D^{L}(i(D)(1-D))_{M}}{1-D}\right)_{M} \\
& +M\left\lfloor\frac{D^{L}(i(D)(1-D))_{M}}{M(1-D)}\right\rfloor_{P / M} \\
& =\left(D^{L} i(D)\right)_{M} \\
& +M\left\lfloor\frac{D^{L}(i(D)(1-D))_{M}}{M(1-D)}\right\rfloor_{P / M}
\end{aligned}
$$

When the last term of (V.22) is zero, $a_{L+1}(D)$ can be obtained by feedforward encoding from $i(D)$, therefore the encoder is feedforward. For difference sequences $i(D)$ with length 1 and symbols in $\{-1,+1\}$ this condition is met. Moreover, due to Gray mapping, the corresponding pairs of binary input sequences have unitary Hamming distance, hence the resulting encoder has finite $d_{E 1}$.

It is well known that in serially concatenated schemes, in order to take advantage of the interleaver gain [19], the inner constituent encoder or modulator must have infinite $d_{E 1}$ [17]. For this reason, when used in SC-CPM schemes, the optimal mapping leads to poor performance and hence a recursive $\mathrm{CPE}$ must be employed. We have thus used the mapping induced by the Rimoldi decomposition, which has this property.

\section{IMPLEMENTATION OF THE OPTIMIZED CODED CPM SCHEMES}

Using the procedures described in the previous sections, SC-CPM and P-CPM schemes have been designed with target capacities of $C_{T}=1 \mathrm{bits} / \mathrm{s} / \mathrm{Hz}, C_{T}=1.5 \mathrm{bits} / \mathrm{s} / \mathrm{Hz}$ and $C_{T}=$ 2 bits/s/Hz.

\section{A. Selection of the modulation schemes}

Fig. 8 and Fig. 9 show the $E_{b} / N_{0}$ values plotted against the CPM complexity for different target capacities. Continuous curves refer to CPM capacity and dashed curves refer to pragmatic capacity.

For REC schemes (Fig. 8) we observe that increasing the complexity beyond 64 yields a negligible gain both in CPM capacity and in pragmatic capacity. Hence, CPM schemes with REC frequency pulse and maximum complexity 64 have been considered.

For RC schemes (Fig. 9), increasing the complexity beyond 64 yields a significant gain both in CPM capacity and in pragmatic capacity at $1.5 \mathrm{bits} / \mathrm{s} / \mathrm{Hz}$ and $2 \mathrm{bits} / \mathrm{s} / \mathrm{Hz}$, hence CPM schemes with higher complexities should be chosen. However, in SC-CPM receivers, the increased complexity would be enhanced by the iterative process, resulting in an impractical solution.

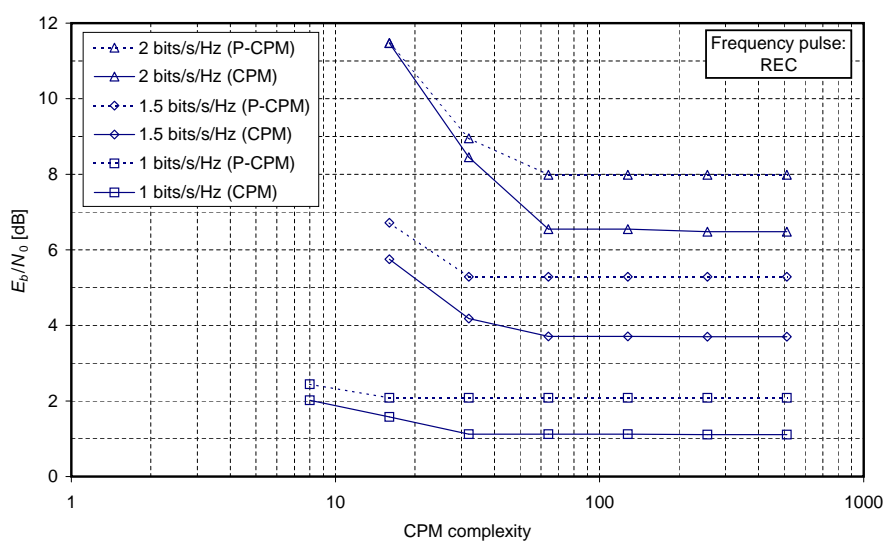

Fig. 8. Energy efficiency of REC CPM schemes.

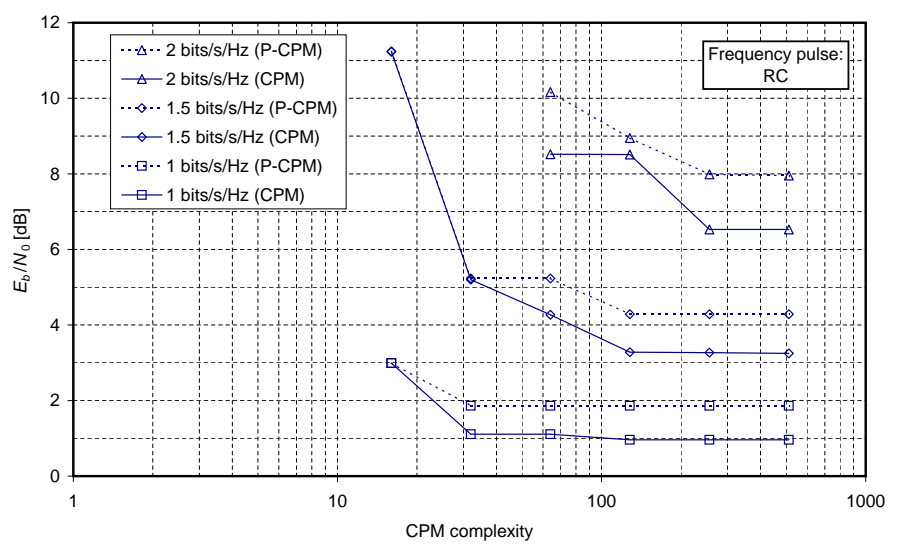

Fig. 9. Energy efficiency of RC CPM schemes.

\section{B. Serially-concatenated schemes}

A serially concatenated CPM scheme consists of the cascade of an outer convolutional encoder connected to the CPM modulator through an interleaver.

As outer encoder, a 4 -state, rate $1 / 2$ systematic recursive convolutional encoder has been chosen. It is shown in Fig. 10. The choice has been dictated by a trade-off between performance and complexity. The encoder output can be punctured 


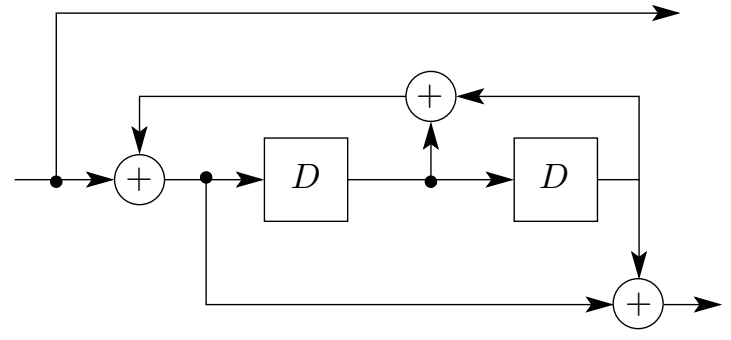

Fig. 10. Convolutional encoder used in SC-CPM schemes.

to achieve rates up to $3 / 4$ while keeping a free distance larger than 2 .

In order to obtain rates larger than $1 / 2$, the output of the convolutional encoder is punctured according to a ratematching algorithm that selects all systematic bits and some coded bits. The puncturing rate is

$$
R_{P}=\frac{N_{O}}{N_{I}} \geq 1
$$

where $N_{O}$ and $N_{I}$ are two integers $\left(N_{I} \leq N_{O} \leq 2 N_{I}\right)$. The puncturing algorithm selects $N_{I}$ coded bits from each block of $N_{O}$ with the following constraint: all systematic bits are selected; some coded bits are selected to achieve the desired rate; the other bits are punctured. The resulting outer code rate is

$$
R_{C C}=\frac{1}{2} \frac{N_{O}}{N_{I}}
$$

The interleaver that connects the punctured outer encoder to the inner CPM modulator is a spread interleaver.

The design of a SC-CPM scheme consists in the choice of the CPM scheme and of the outer encoder based on the desired capacity and on design constraints (e.g., the maximum affordable complexity of the CPM scheme).

Example 6.1: We describe the design of a SC-CPM scheme with target capacity $C_{T}=1.5 \mathrm{bits} / \mathrm{s} / \mathrm{Hz}, \mathrm{REC}$ frequency pulse and maximum CPM complexity of 64 edges per CPM input bit. As for SC-CPM schemes, the parameters that maximize $C_{\mathrm{CPM}}$ must be chosen, from Tab. I] Moreover, since the maximum CPM complexity is 64 , we must restrict the search to the column set labelled 64. Next, in the column labelled $C$, we look for the value closest to the target capacity. We find that the capacity value closest to $C_{T}$ is 1.51 and the corresponding CPM parameters are $m=2, h=1 / 5, L=2$. Such scheme exhibits a symbol rate $R_{s}=1.18$ and a corresponding bit rate $R_{b}=m R_{s}=2.36$. The scheme of the CPM modulator corresponding to these parameters is shown in Fig. 11

In order to obtain a target capacity $C_{T}=1.5 \mathrm{bits} / \mathrm{s} / \mathrm{Hz}$, the outer code rate must be set to

$$
R_{C C}=\frac{N_{O}}{2 N_{I}}=\frac{C_{T}}{R_{b}}=0.636
$$

therefore

$$
\frac{N_{O}}{N_{I}}=\frac{2 C_{T}}{R_{b}}=1.2712 \simeq \frac{75}{59} .
$$

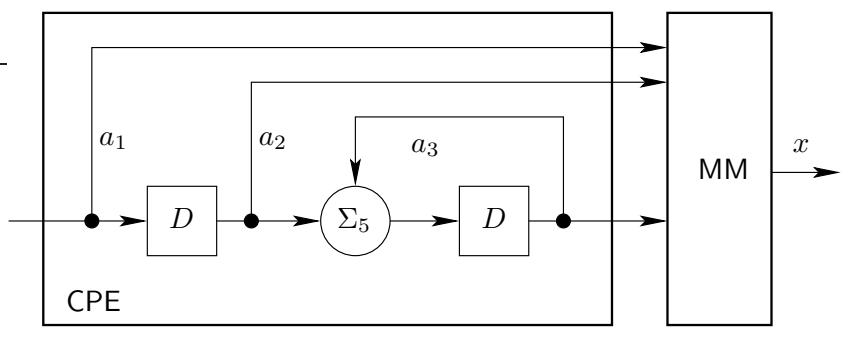

Fig. 11. Scheme of the CPM modulator with $m=2, h=1 / 5, L=2$.

The final step consists in choosing two integers for $N_{O}$ and $N_{I}$ in order to approximate with sufficient precision this ratio.

The resulting design parameters for all considered target capacities are shown in Tab. V.

\section{Pragmatic schemes}

A pragmatic CPM scheme consists of the cascade of a SCCC encoder and a CPM modulator.

The chosen encoder is the SCCC encoder described in [12]. The constituent convolutional encoders used in such scheme are the 4-state convolutional encoders shown in Fig. 10 . The outer convolutional encoder is punctured to a rate $2 / 3$.

The inner encoder is punctured using a carefully designed rate-matching algorithm that selects all the systematic bits and some coded bits in order to achieve the desired overall rate.

The interleaver that connects the punctured outer encoder to the inner encoder is a spread interleaver.

Example 6.2: We show how to design a P-CPM scheme with target capacity of 2 bits/s/Hz, RC frequency pulse and maximum CPM complexity of 64 edges per CPM input bit. For P-CPM schemes, the inner CPM modulation parameters that maximize $C_{\mathrm{P}-\mathrm{CPM}}$ must be chosen. Therefore, Tab. IV must be considered. Moreover, since the maximum CPM complexity is 64 , we must restrict the search to the column set labelled 64 . Next, in column labelled $C$ we look for the value closest to the target capacity. We find that the capacity value closest to $C_{T}$ is 2.00 and the corresponding CPM parameters are $m=2, h=$ $1 / 8, L=2$. Such scheme exhibits a symbol rate $R_{s}=1.18$ and a corresponding bit rate $R_{b}=m R_{s}=2.36$. The scheme of the CPM modulator corresponding to these parameters is shown in Fig. 12 .

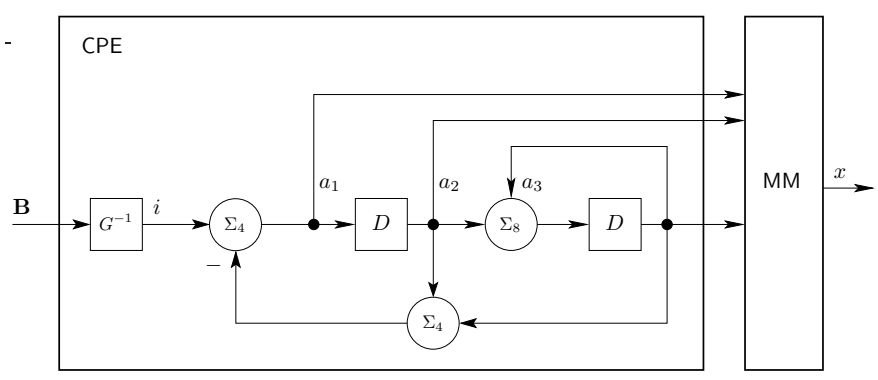

Fig. 12. Scheme of the CPM modulator with $m=2, h=1 / 8, L=2$.

In order to obtain a target capacity $C_{T}=2 \mathrm{bits} / \mathrm{s} / \mathrm{Hz}$, the outer code rate must be 


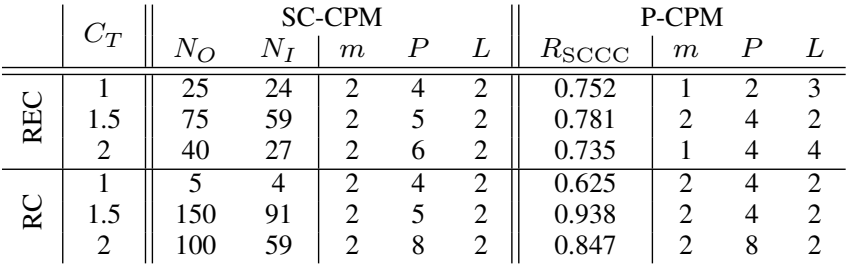

TABLE V

PARAMETERS OF THE OPTIMIZED SC-CPM AND P-CPM SCHEMES YIELDING 1.0, 1.5 AND $2.0 \mathrm{BITS} / \mathrm{s} / \mathrm{HZ}$

$$
R_{\mathrm{SCCC}}=\frac{C_{T}}{R_{b}}=0.847
$$

Tab. $\mathrm{V}$ summarizes the parameters of the designed schemes.

\section{RESULTS}

The performance of the designed coding schemes reported in Tab. $\mathrm{V}]$ has been assessed through simulation. For each scheme, the CPM capacity and the pragmatic capacity have been estimated. The obtained results are shown in Fig. 13 for REC frequency pulses and Fig. 14 for RC frequency pulses.

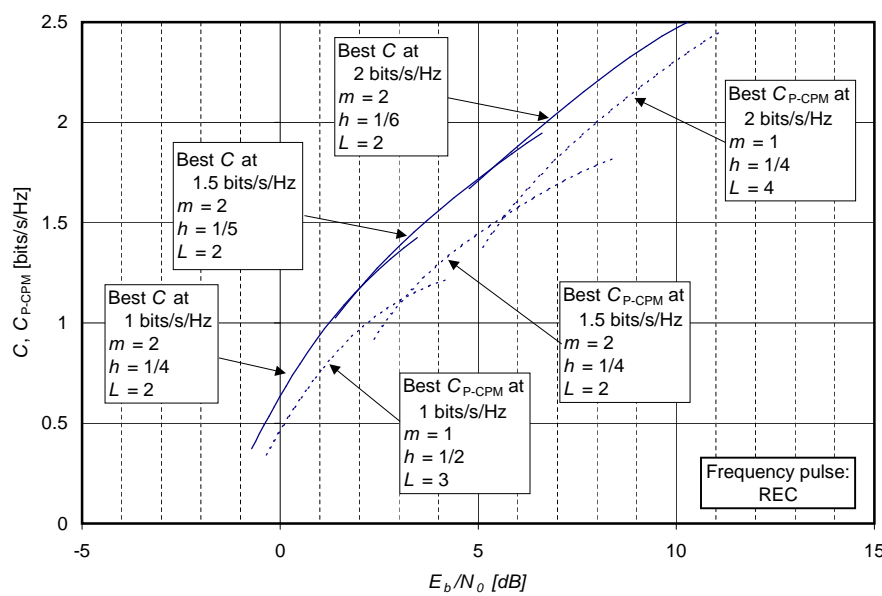

Fig. 13. CPM capacity and pragmatic capacity of the selected CPM schemes with complexity $\mathcal{Y} \leq 64$. The frequency pulse is REC.

In both the P-CPM and the SC-CPM case, an outer information word length of $K=9840$ bits has been considered and the code rates have been set according to Tab. V The number of decoding iterations has been set to 10 .

The information rates $I_{\mathrm{SC}-\mathrm{CPM}}$ (resp. $I_{\mathrm{P}-\mathrm{CPM}}$ ) of the SC-CPM (resp. P-CPM) schemes has been evaluated through simulation by computing the mutual information between the information bits at the input of the channel encoder and the soft outputs of the channel decoder. Fig. 15. Fig. 16 and Fig. 17 show the obtained information rates for REC frequency pulse and complexity $\mathcal{Y} \leq 64$. We observe that, for a capacity of $1 \mathrm{bits} / \mathrm{s} / \mathrm{Hz}$, the pragmatic scheme achieves the desired information rate at a lower $E_{b} / N_{0}$ than the SC-CPM scheme, and at $1 \mathrm{~dB}$ from the pragmatic capacity. For capacities of 1.5 bits/s/Hz and 2 bits $/ \mathrm{s} / \mathrm{Hz}$, the SC-CPM scheme achieves the

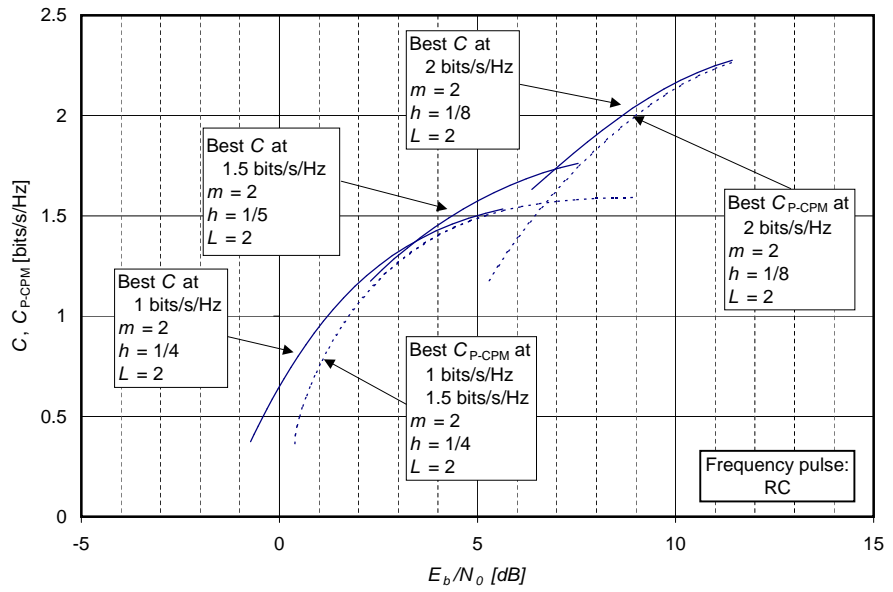

Fig. 14. CPM capacity and pragmatic capacity of the selected CPM schemes with complexity $\mathcal{Y} \leq 64$. The frequency pulse is RC.

desired information rate at a lower $E_{b} / N_{0}$ than the P-CPM scheme.

The best SC-CPM and P-CPM for the chosen spectral efficiencies have been simulated over an AWGN channel so as to obtain accurate estimates of the bit and frame error probabilities at very low values. The results are shown in Fig. 18 We observe that, at $1 \mathrm{bits} / \mathrm{s} / \mathrm{Hz}$, the P-CPM scheme, while featuring a lower complexity (see Tab. VI), exhibits a $0.5 \mathrm{~dB}$ gain over the SC-CPM scheme at FER $=10^{-4}$. At higher spectral efficiencies, the SC-CPM scheme exhibits a gain of more than $1 \mathrm{~dB}$ over the P-CPM scheme.

Fig. 19 shows similar results for RC frequency pulses. The SC-CPM schemes feature a $0.3 \mathrm{~dB}$ gain over the P-CPM schemes at $1 \mathrm{bits} / \mathrm{s} / \mathrm{Hz}$ and $2 \mathrm{bits} / \mathrm{s} / \mathrm{Hz}$, while at $1.5 \mathrm{bits} / \mathrm{s} / \mathrm{Hz}$ the gain of the SC-CPM scheme is about $1 \mathrm{~dB}$. In both frequency pulses and all spectral efficiencies, no error floors have been observed.

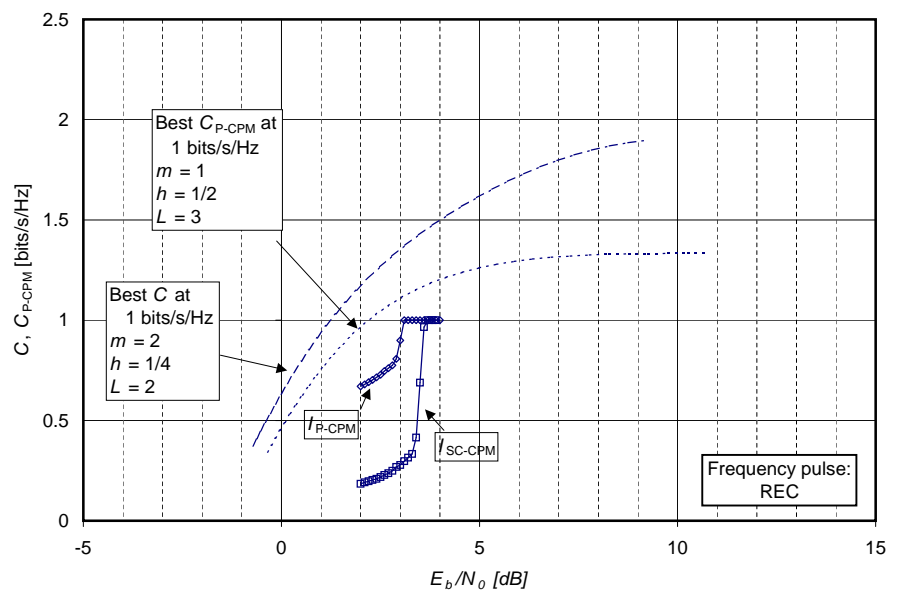

Fig. 15. Information rates of P-CPM and SC-CPM coded systems at 1 bits/s/Hz. $\mathcal{Y}=64$, REC frequency pulse.

A comparison of REC and RC pragmatic schemes is shown in Fig. 20. We observe that the RC scheme performs better than REC scheme at $1 \mathrm{bits} / \mathrm{s} / \mathrm{Hz}$ and $1.5 \mathrm{bits} / \mathrm{s} / \mathrm{Hz}$. At 2 bits $/ \mathrm{s} / \mathrm{Hz}$, the hierarchy is reversed. At 2 bits $/ \mathrm{s} / \mathrm{Hz}, \mathrm{REC}$ schemes feature a gain of roughly $0.6 \mathrm{~dB}$ over the $\mathrm{RC}$ schemes at $\mathrm{FER}=10^{-4}$. 


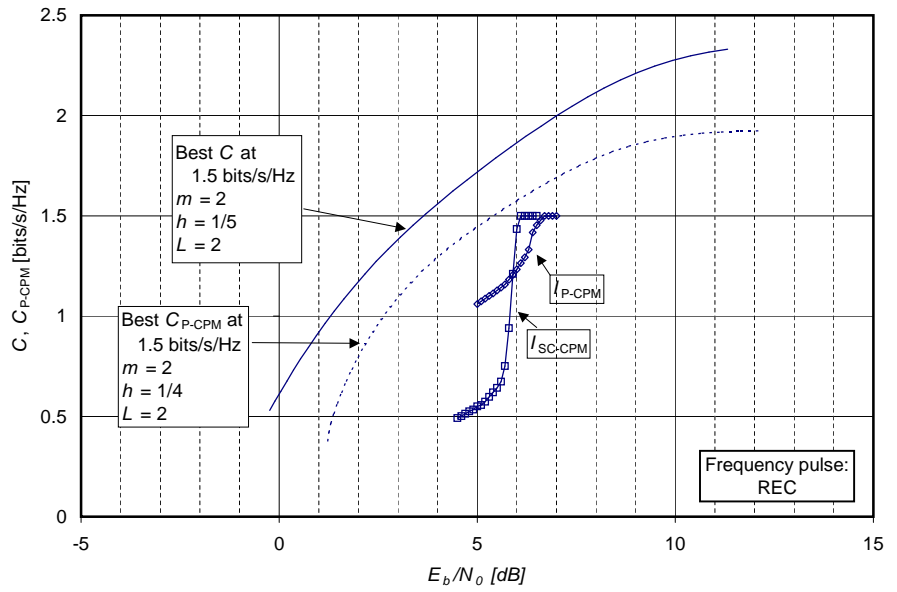

Fig. 16. Information rates of P-CPM and SC-CPM coded systems at 1.5 bits/s/Hz. $\mathcal{Y}=64$, REC frequency pulse.

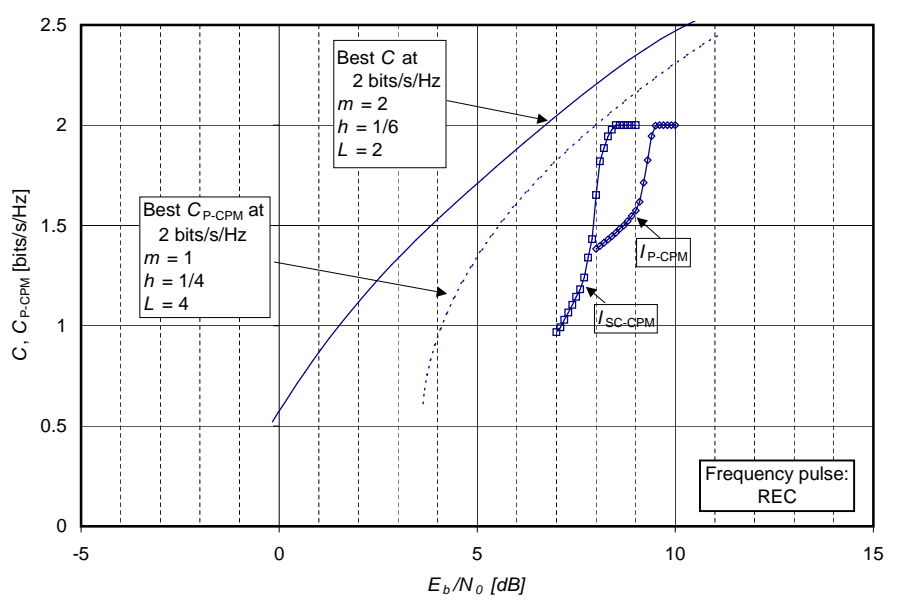

Fig. 17. Information rates of P-CPM and SC-CPM coded systems at 2 bits/s/Hz. $\mathcal{Y}=64$, REC frequency pulse.

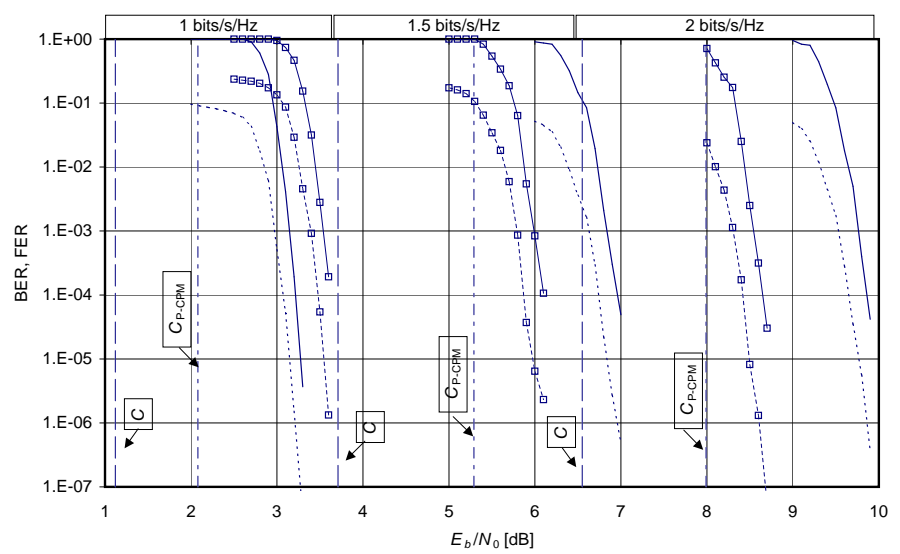

Fig. 18. Error rates of P-CPM (curves without markers) and SC-CPM (curves with markers) coded systems. $\mathcal{Y}=64$, REC frequency pulse.

In order to perform a fair comparison, the decoding complexity must be kept into account. Using definitions (II.3) and (II.4), the decoding complexities of the two schemes have been computed. For the P-CPM scheme, the complexity of the MHOMS [12] binary decoder has been computed as follows:

$$
\mathcal{Y}_{\mathrm{SCCC}}=2 N_{i t}\left(N_{s o}+\frac{3}{2} N_{s i}\right)
$$

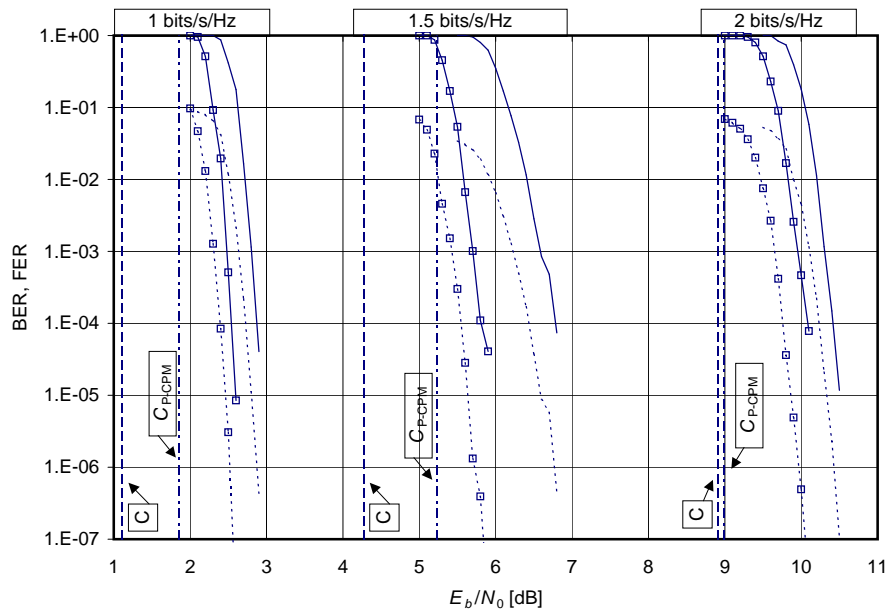

Fig. 19. Error rates of P-CPM (curves without markers) and SC-CPM (curves with markers) coded systems. $\mathcal{Y}=64, \mathrm{RC}$ frequency pulse.

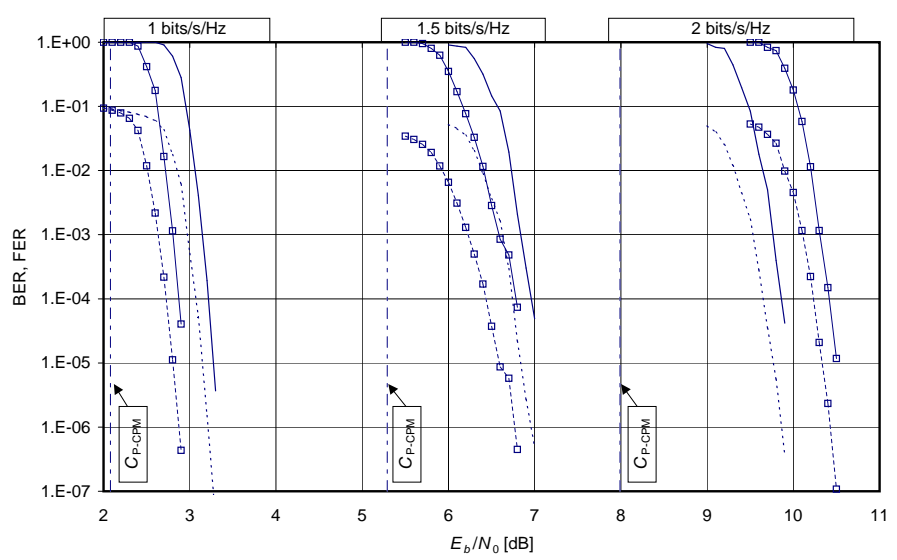

Fig. 20. Comparison of REC (curves without markers) and RC (curves with markers) P-CPM schemes with $\mathcal{Y} \leq 64$.

where the factor $3 / 2$ derives from the outer code rate of the MHOMS scheme, which is $2 / 3$. Results are shown in Tab. VI we observe that the complexity of the SC-CPM scheme is always larger than that of the P-CPM scheme. We define the complexity ratio $R_{\mathcal{Y}}=\mathcal{Y}_{\mathrm{SC}-\mathrm{CPM}} / \mathcal{Y}_{\mathrm{P}-\mathrm{CPM}}$ : its value is larger than 2 and exceeds 3 for a 1 bits $/ \mathrm{s} / \mathrm{Hz}$ capacity with REC frequency pulse. Higher coding gains could be achieved increasing the P-CPM complexity, thus reducing the performance gap with respect to SC-CPM schemes.

\begin{tabular}{|c|c|c|c|c|c|c|}
\hline & \multirow{2}{*}{ Capacity } & \multicolumn{3}{|c|}{$P-C P M$} & \multirow{2}{*}{$\begin{array}{l}S C-C P M \\
\mathcal{Y}_{S C-C P M}\end{array}$} & \multirow{2}{*}{$\begin{array}{l}\text { Ratio } \\
R_{\mathcal{Y}}\end{array}$} \\
\hline & & $\mathcal{Y}_{S C C C}$ & $\mathcal{Y}_{C P M}$ & $\mathcal{Y}_{P-C P M}$ & & \\
\hline & 1 & 200 & 21 & 221 & 694 & 3.14 \\
\hline 夏 & 1.5 & 200 & 41 & 241 & 709 & 2.94 \\
\hline & 2 & 200 & 87 & 287 & 728 & 2.54 \\
\hline & 1 & 200 & 51 & 251 & 592 & 2.36 \\
\hline$\underset{\sim}{ }$ & 1.5 & 200 & 34 & 234 & 565 & 2.41 \\
\hline & 2 & 200 & 76 & 276 & 733 & 2.66 \\
\hline
\end{tabular}

TABLE VI

COMPLEXITY OF P-CPM AND SC-CPM SCHEMES. $\mathcal{Y}=64$.

\section{CONCLUSIONS}

The pragmatic approach to coded continuous-phase modulation (CPM) has been proposed as a capacity-approaching low- 
complexity alternative to the serially-concatenated CPM (SC$\mathrm{CPM}$ ) coding scheme. After performing a selection of the best spectrally-efficient CPM modulations to be embedded into SCCPM schemes, the pragmatic capacity of CPM modulations has been evaluated and optimized through a careful design of the mapping between input bits and CPM waveforms. The so obtained schemes have been cascaded with an outer serially-concatenated convolutional code to form a pragmatic coded-modulation system. The resulting schemes have been shown to exhibit performance close to the CPM capacity without requiring iterations between the outer decoder and the CPM demodulator. As a result, the receiver exhibits reduced complexity and increased flexibility due to the separation of the demodulation and decoding functions.

\section{ACKNOWLEDGEMENTS}

This work has been supported by Regione Piemonte under Contract E4.

\section{REFERENCES}

[1] J. B. Anderson, T. Aulin, and C.-E. Sundberg, Digital Phase Modulation New York, London: Plenum Press, 1986.

[2] B. E. Rimoldi, "A decomposition approach to CPM," IEEE Transactions on Information Theory, vol. 34, pp. 260-270, March 1988

[3] S. Benedetto, D. Divsalar, G. Montorsi, and F. Pollara, "Serial concatenation of interleaved codes: Performance analysis, design adn iterative decoding," IEEE Transactions on Information Theory, vol. 44, pp. 909 926, May 1998.

[4] P. Moqvist and T. M. Aulin, "Serially concatenated continuous phase modulation with iterative decoding," IEEE Transactions on Communications, vol. 49, pp. 1901-1915, November 2001.

[5] K. R. Narayanan and G. L. Stüber, "Performance of trellis-coded CPM with iterative demodulation and decoding," IEEE Transactions on Communications, vol. 49, pp. 676-687, April 2001.

[6] K. R. Narayanan, I. Altunbas, and R. S. Narayanaswami, "Design of serial concatenated MSK schemes based on density evolution," IEEE Transactions on Communications, vol. 51, pp. 1283-1295, August 2003.

[7] D. Arnold, H.-A. Loeliger, P. A. Vontobel, A. Kavčić, and W. Zeng, "Simulation-based computation of information rates for channels with memory," IEEE Transactions on Information Theory, vol. 52, pp. 3498 3508, August 2006

[8] K. Padmanabhan, S. Ranganathan, S. P. Sundaravaradhan, and O. M. Collins, "General CPM and its capacity," in Proceedings of the International Symposium on Information Theory, Adelaide, AU, September 2005, pp. 750-754.

[9] A. J. Viterbi, J. K. Wolf, E. Zehavi, and R. Padovani, "A pragmatic approach to trellis-coded modulation," IEEE Communications Magazine, vol. 27 , no. 7 , pp. 11-19, July 1989 .

[10] E. Zehavi, "8-psk trellis codes ofr a rayleight channel," IEEE Transactions on Communications, vol. 40, no. 5, pp. 873-884, May 1992.

[11] G. Caire, G. Taricco, and E. Biglieri, "Bit-interleaved coded modulation," IEEE Transactions on Information Theory, vol. 44, pp. 927-946, May 1998.

[12] S. Benedetto, R. Garello, G. Montorsi, C. Berrou, C. Douillard, D. Giancristofaro, A. Ginesi, L. Giugno, and M. Luise, "MHOMS: high-speed ACM modem for satellite applications," IEEE Wireless Communications, pp. 66-77, April 2005

[13] S. Benedetto, G. Montorsi, A. Perotti, and A. Tarable, "A pragmatic approach to coded continuous-phase modulation," in Workshop on Information Theory and Applications, San Diego (CA), USA, January 2007.

[14] - "Optimization of CPM pragmatic capacity" in IEEE Global Communications Conference, Washington (DC), USA, November 2007.

[15] M. R. Shane and R. D. Wesel, "Parallel concatenated turbo codes for continuous phase modulation," in IEEE Wireless Communications and Networking Conference, Chicago (IL), USA, September 2000, pp. 147152.

[16] W. Zhao and G. B. Giannakis, "Reduced complexity receivers for layered space-time CPM," IEEE Transactions on Wireless Communications, vol. 4, no. 2, pp. 574-582, March 2005.
[17] S. Benedetto, D. Divsalar, G. Montorsi, and F. Pollara, "Soft-input softoutput modules for the construction and distributed iterative decoding of code networks," European Transactions on Telecommunications, vol. 9 pp. 155-172, March 1998.

[18] W. Press, S. Teukolsky, W. Vetterling, and B. Flannery, Numerical Recipes in $C, 2$ nd ed. Cambridge, UK: Cambridge University Press, 1992.

[19] S. Benedetto and G. Montorsi, "Unveiling turbo codes: Some results on parallel concatenated coding schemes," IEEE Transactions on Information Theory, vol. 42, no. 2, pp. 409-428, March 1996.

\section{APPENDIX I \\ PROOF OF THEOREM 5.1}

First, we revise some properties of the CPM trellis, for a binary CPM scheme.

An error event generated by a given difference sequence $\mathbf{b}=\left(b_{1}, \ldots, b_{\Delta}\right) \in\{-1,0,1\}^{\Delta}$, is a pair of trellis paths with length $\Delta+L-1$ trellis steps, which diverge at, say, time 0 and merge back together at time $\Delta+L-1$. For a given value of $\mathbf{b}$, there exist several different error events, corresponding to all possible choices of the variables:

$$
\beta_{0}, a_{-L+2}, \ldots, a_{0}, a_{1}^{(1)}, \ldots, a_{\Delta}^{(1)}, a_{\Delta+1}, \ldots, a_{\Delta+L-1}
$$

and

$$
\beta_{0}, a_{-L+2}, \ldots, a_{0}, a_{1}^{(2)}, \ldots, a_{\Delta}^{(2)}, a_{\Delta+1}, \ldots, a_{\Delta+L-1}
$$

where $\beta_{0}, a_{-L+2}, \ldots, a_{0}$ determine the starting state,

$$
a_{n}^{(1)}=\left\{\begin{array}{cc}
1 & b_{n}=1 \\
0 & b_{n}=-1 \\
a_{n} & b_{n}=0
\end{array}\right.
$$

and

$$
a_{n}^{(2)}=\left\{\begin{array}{cc}
0 & b_{n}=1 \\
1 & b_{n}=-1 \\
a_{n} & b_{n}=0
\end{array},\right.
$$

and $a_{\Delta+1}, \ldots, a_{\Delta+L-1}$ determine the final state of the error event. If two trellis edges belong to the same trellis section of the error event, we call them an edge pair of the error event.

Now, we build the graph $\mathcal{G}(\mathbf{b})=(\mathcal{V}, \mathcal{E})$ as described in Sect. $\mathrm{V}-\mathrm{B}$ two vertices are adjacent if and only if the corresponding trellis edges do not have the same starting state and constitute an edge pair of an error event generated by $\mathbf{b}$. The graph $\mathcal{G}(\mathbf{b})$ has $\mathcal{N}(\mathbf{b})$ connected components, denoted $\mathcal{C}_{0}, \ldots, \mathcal{C}_{\mathcal{N}(\mathbf{b})-1}$, where $\mathcal{C}_{i}=\left(\mathcal{V}_{i}, \mathcal{E}_{i}\right)$, whose properties will be investigated hereafter.

For an integer amount $\gamma$, the $\gamma$-rotation transforms the trellis edge $(\alpha, \beta)$ into the trellis edge $\left(\alpha,(\beta+\gamma)_{P}\right)$. The image of a set of edges through the $\gamma$-rotation is the set of images of the edges belonging to the set through the $\gamma$-rotation. The following proposition holds:

Proposition 1.1: For any $\gamma$, the $\gamma$-rotation transforms the set $\mathcal{V}_{i}$, corresponding to a component $\mathcal{C}_{i}$ of $\mathcal{G}(\mathbf{b})$, into a set $\mathcal{V}_{j}$, corresponding to another component $\mathcal{C}_{j}$ (even, possibly, the same)

Proof: Two trellis edges $(\alpha, \beta)$ and $\left(\alpha^{\prime}, \beta^{\prime}\right)$ are in the same component $\mathcal{C}_{i}$ if and only if in the graph there is a length$\kappa$ path,

$$
\left(\alpha^{(1)}, \beta^{(1)}\right) \rightarrow\left(\alpha^{(2)}, \beta^{(2)}\right) \rightarrow \cdots \rightarrow\left(\alpha^{(\kappa)}, \beta^{(\kappa)}\right)
$$


with $\left(\alpha^{(1)}, \beta^{(1)}\right)=(\alpha, \beta)$ and $\left(\alpha^{(\kappa)}, \beta^{(\kappa)}\right)=\left(\alpha^{\prime}, \beta^{\prime}\right)$. This means that, for $i=1, \ldots, \kappa-1,\left(\alpha^{(i)}, \beta^{(i)}\right)$ and $\left(\alpha^{(i+1)}, \beta^{(i+1)}\right)$ have different starting states and form an edge pair of an error event generated by $\mathbf{b}$.

Now, notice that, given an error event generated by $\mathbf{b}$, by changing $\beta_{0}$ in $\left(\right.$ I.1) and $\left(\right.$ I.2 into $\left(\beta_{0}+\gamma\right)_{P}$, we obtain another error event generated by $\mathbf{b}$, whose edge pairs are in one-to-one correspondence through $\gamma$-rotation with the edge pairs of the original error event. For this reason, in the graph there is the path

$$
\left(\alpha^{(1)}, \beta_{\gamma}^{(1)}\right) \rightarrow\left(\alpha^{(2)}, \beta_{\gamma}^{(2)}\right) \rightarrow \cdots \rightarrow\left(\alpha^{(\kappa)}, \beta_{\gamma}^{(\kappa)}\right)
$$

where $\beta_{\gamma}^{(i)} \triangleq\left(\beta^{(i)}+\gamma\right)_{P}$.

Thus, by definition, $\left(\alpha,(\beta+\gamma)_{P}\right)$ and $\left(\alpha^{\prime},\left(\beta^{\prime}+\gamma\right)_{P}\right)$ belong to the same component $\mathcal{C}_{j}$. For the arbitrariness of $(\alpha, \beta)$ and $\left(\alpha^{\prime}, \beta^{\prime}\right)$, we have that the image of $\mathcal{V}_{i}$ through $\gamma$-rotation is contained in $\mathcal{V}_{j}$.

Finally, notice that, with the same argument, the image of $\mathcal{V}_{j}$ through $(P-\gamma)$-rotation is contained in $\mathcal{V}_{i}$. From this fact, and the injectivity of rotations, we deduce that the image of $\mathcal{V}_{i}$ through $\gamma$-rotation is equal to $\mathcal{V}_{j}$.

From (I.1) and (I.2), we can deduce a set of rules for a pair of trellis edges to be in the same component, in the following way:

- We fix a trellis section inside the error event. The error event has length $\Delta+L-1$ trellis sections, but the first is not to be considered, thus we have $\Delta+L-2$ possible choices.

- We consider the constraints imposed on an edge pair of the error event at that trellis section.

We derive two types of rules, according to the trellis section we are considering.

Type-I rules: They correspond to the last $L$ trellis sections of the error event. They give the following edge pairs, for $j=\Delta-L+1, \ldots, \Delta$ :

$$
\left(\alpha_{j}^{(1)},\left(\beta_{\Delta+L-1}-Q w_{H}\left(\alpha_{j}^{(1)}\right)\right)_{P}\right)
$$

and

$$
\left(\alpha_{j}^{(2)},\left(\beta_{\Delta+L-1}-Q w_{H}\left(\alpha_{j}^{(2)}\right)\right)_{P}\right),
$$

where $\alpha_{j}^{(i)}=\left(a_{j}^{(i)}, \ldots, a_{j+L-1}^{(i)}\right), a_{j}^{(i)}=a_{j}$ if $j<1$ or $j>\Delta$, and

$$
\beta_{\Delta+L-1}=\left(\beta_{0}+Q \sum_{i=-L+2}^{\Delta} a_{i}^{(1)}\right)_{P} .
$$

The above relation holds because $\sum_{i=1}^{\Delta} a_{i}^{(1)}=\sum_{i=1}^{\Delta} a_{i}^{(2)}$ $\bmod P$.

Type-II rules: They correspond to the $\Delta-2$ trellis sections of the error event from the second to the $(\Delta-1)$-th one. They give the following edge pairs, for $j=-L+3, \ldots, \Delta-L$ :

$$
\left(\alpha_{j}^{(1)}, \beta_{j+L-1}^{(1)}\right)
$$

and

$$
\left(\alpha_{j}^{(2)}, \beta_{j+L-1}^{(2)}\right)
$$

where $\alpha_{j}^{(i)}=\left(a_{j}^{(i)}, \ldots, a_{j+L-1}^{(i)}\right), a_{j}^{(i)}=a_{j}$ if $j<1$ or $j>\Delta$, and

$$
\beta_{j+L-1}^{(i)}=\left(\beta_{0}+Q \sum_{k=-L+2}^{j} a_{k}^{(i)}\right)_{P} .
$$

Notice that, if $\Delta=2$, there are no type-II rules.

The following lemma will be the key to prove Theorem 5.1

Lemma 1.2: Consider the sets of trellis edges:

$$
\widetilde{\mathcal{V}}_{i}=\left\{\left(\alpha,\left(i-Q w_{H}(\alpha)\right)_{P}\right): \alpha \in\{0,1\}^{L}\right\},
$$

for $i=0, \ldots, P-1$. Each of these sets is entirely contained in one connected component of the graph $\mathcal{G}(\mathbf{b})$.

Proof: We prove by induction that the set of type-I rules implies the lemma, starting from the last rule (i.e., $j=\Delta$ in (I.5) and (I.6) and going backwards.

Suppose that the last $d$ rules $(d<L)$ imply that the set of edges of the form:

$\widetilde{\mathcal{V}}_{\beta_{\Delta+L-1}, d}(\mathbf{x})=\left\{\left([\mathbf{d}, \mathbf{x}],\left(\beta_{\Delta+L-1}-Q w_{H}([\mathbf{d}, \mathbf{x}])\right)_{P}\right), \mathbf{d} \in\{0,1\}^{d}\right\}$

for a fixed length- $(L-d)$ vector $\mathbf{x}=\left(x_{1}, \ldots, x_{L-d}\right)$, is entirely contained in one connected component. This is true for $d=1$, since $b_{\Delta} \neq 0$.

Now, again because $b_{\Delta} \neq 0$, the last-but- $(d+1)$ rule pairs edges like $\mathbf{d}^{\prime} x_{1} \ldots x_{L-d}$ and $\mathbf{d}^{\prime \prime} \overline{x_{1}} \ldots x_{L-d}$, for suitable length- $d$ vectors $\mathbf{d}^{\prime}$ and $\mathbf{d}^{\prime \prime}$, where $\bar{x}$ is the complement of $x$. This implies that

$$
\tilde{\mathcal{V}}_{\beta_{\Delta+L-1}, d+1}(\mathbf{x})=\widetilde{\mathcal{V}}_{\beta_{\Delta+L-1}, d}([0, \mathbf{x}]) \cup \widetilde{\mathcal{V}}_{\beta_{\Delta+L-1}, d}([1, \mathbf{x}]),
$$

for a fixed length- $(L-d-1)$ vector $\mathbf{x}$, is entirely contained in one connected component.

By induction up to $d=L$, we have proved that, for all possible values of $\beta_{\Delta+L-1}, \widetilde{\mathcal{V}}_{\beta_{\Delta+L-1}}=\widetilde{\mathcal{V}}_{\beta_{\Delta+L-1}, L}$ is entirely contained in one connected component.

Proof: [Of Theorem 5.1] If $\Delta(\mathbf{b})=2$, then there are only type-I rules, and the components of $\mathcal{G}(\mathbf{b})$ satisfy, for $i=0, \ldots, P-1$ :

$$
\mathcal{V}_{i}=\widetilde{\mathcal{V}}_{i}
$$

and the theorem is proved.

If $\Delta(\mathbf{b})>2$, instead, there are also type-II rules. Consider, in particular, the type-II rule with $j=\Delta-L$ in (I.7) and (I.8). It gives the edge pair:

$$
\left(\alpha_{\Delta-L}^{(1)}, \beta_{\Delta-1}^{(1)}\right)
$$

and

$$
\left(\alpha_{\Delta-L}^{(2)}, \beta_{\Delta-1}^{(2)}\right)
$$

But the above transitions belong to $\widetilde{\mathcal{V}}_{\left(\beta_{\Delta+L-1}-Q\right)_{P}}$ and to $\widetilde{\mathcal{V}}_{\beta_{\Delta+L-1}}$. Then, these two sets are merged by the type-II rule with $j=\Delta-L$. Consider the component $\mathcal{C}_{1}$ of $\mathcal{G}(\mathbf{b})$ that contains these sets and let $\mathcal{V}_{1}$ be its vertex set. Prop. 1.1 implies that a $Q$-rotation transforms $\mathcal{V}_{1}$ into itself. But then, $\mathcal{C}_{1}$ also contains $\widetilde{\mathcal{V}}_{\left(\beta_{\Delta+L-1}-l Q\right)_{P}}$, for every integer $l$. Since $Q$ and $P$ are 3 , we deduce that $\mathcal{C}_{1}$ contains all $\widetilde{\mathcal{V}}_{i}$ 's and thus is the only component of $\mathcal{G}(\mathbf{b})$. This concludes the proof. 
APPENDIX II

PROOF OF THEOREM 5.2

To prove Theorem 5.2, we first notice that Prop. 1.1 holds for a $1 \mathrm{CPM}$ scheme also, since in the proof, the hypothesis of binary input symbols is not used.

Also, there are two types of rules, which are the extension to the 1 case of the two types introduced in the previous sections.

Type-I rules: They correspond to the last $L$ trellis sections of the error event. They give the following edge pairs, for $j=\Delta-L+1, \ldots, \Delta$ :

$$
\left(\alpha_{j}^{(1)},\left(\beta_{\Delta+L-1}-Q \sum_{i=0}^{L-1} \alpha_{j+i}^{(1)}\right)_{P}\right)
$$

and

$$
\left(\alpha_{j}^{(2)},\left(\beta_{\Delta+L-1}-Q \sum_{i=0}^{L-1} \alpha_{j+i}^{(2)}\right)_{P}\right),
$$

where $\alpha_{j}^{(i)}=\left(a_{j}^{(i)}, \ldots, a_{j+L-1}^{(i)}\right), a_{j}^{(i)}=a_{j}$ if $j<1$ or $j>\Delta$, and

$$
\beta_{\Delta+L-1}=\left(\beta_{0}+Q \sum_{i=-L+2}^{\Delta} a_{i}^{(1)}\right)_{P} .
$$

The above relation holds because $\sum_{i=1}^{\Delta} a_{i}^{(1)}=\sum_{i=1}^{\Delta} a_{i}^{(2)}$ $\bmod P$.

Type-II rules: They correspond to the $\Delta-2$ trellis sections of the error event from the second to the $(\Delta-1)$-th one. They give the following edge pairs, for $j=-L+3, \ldots, \Delta-L$ :

$$
\left(\alpha_{j}^{(1)}, \beta_{j+L-1}^{(1)}\right)
$$

and

$$
\left(\alpha_{j}^{(2)}, \beta_{j+L-1}^{(2)}\right)
$$

where $\alpha_{j}^{(i)}=\left(a_{j}^{(i)}, \ldots, a_{j+L-1}^{(i)}\right), a_{j}^{(i)}=a_{j}$ if $j<1$ or $j>\Delta$, and

$$
\beta_{j+L-1}^{(i)}=\left(\beta_{0}+Q \sum_{k=-L+2}^{j} a_{k}^{(i)}\right)_{P} .
$$

Notice that, if $\Delta=2$, there are no type-II rules.

The following lemma is the extension of Lemma 1.2 to the 1 case.

Lemma 2.1: Let $\mathbf{b}$ be a difference sequence of length $\Delta$ with $b_{\Delta}= \pm 1$. Consider the sets:

$$
\widetilde{\mathcal{C}}_{i}=\left\{\left(\alpha,\left(i-Q \sum_{i=1}^{L} \alpha_{i}\right)_{P}\right): \alpha \in\{0, \ldots, M-1\}^{L}\right\}_{(\text {II. } 5)}
$$

for $i=0, \ldots, P-1$. Each of these sets is entirely contained in one connected component of the graph $\mathcal{G}(\mathbf{b})$.

Proof: By induction on the set of type-I rules. It is the straightforward extension of Lemma 1.2.

Finally, the proof of Theorem 5.2 follows the proof of Theorem 5.1, by noticing that if $\Delta(\mathbf{b})=2$, then there are only type-I rules, while if $\Delta(\mathbf{b})>2$ there are also type-II rules, that cause the graph $\mathcal{G}(\mathbf{b})$ to be connected. 